\title{
Katalytische Olefinpolymerisation in wässerigen Systemen
}

\author{
Stefan Mecking,* Anke Held und Florian M. Bauers
}

\begin{abstract}
Übergangsmetall-katalysierte Umsetzungen in wässerigen Medien sind in den vergangenen zwei Jahrzehnten intensiv untersucht worden und haben sich zu einem umfangreichen Gebiet entwickelt. Die große Mehrzahl der Arbeiten befasste sich mit der Synthese niedermolekularer Verbindungen. Dabei ist Wasser insbesondere für Polymerisationsreaktionen ein attraktives Reaktionsmedium. Zum Beispiel werden Emulsions- und Suspensionspolymerisationen in Wasser heute im großen Maßstab mittels (nichtkatalytischer) radikalischer Verfahren durchgeführt. Als Produkt können Polymerlatices erhalten werden, d.h. stabile wässerige Dispersionen von
\end{abstract}

\begin{abstract}
Polymerpartikeln mit Größen von 50 bis $1000 \mathrm{~nm}$. Solche Latices haben ein außergewöhnliches Eigenschaftsprofil. Die Verwendung von Wasser als Dispersionsmedium ist zudem besonders umweltfreundlich. Im Vergleich zu diesen radikalischen Reaktionen haben katalytische Polymerisationen von Olefinen in Wasser weniger Aufmerksamkeit gefunden. In jüngster Zeit wurden jedoch erhebliche Fortschritte erzielt. Verschiedenste hochmolekulare Polymere, von amorphen oder teilkristallinen Polyolefinen bis zu polar substituierten hydrophilen Materialien, wurden mittels katalytischer Polymerisation olefinischer Monomere in Wasser hergestellt. Polymerlatices sind
\end{abstract}

ausgehend von gut verfügbaren Monomeren zugänglich. Bei ihrer Synthese wurden bereits katalytische Aktivitäten bis $10^{5}$ mol umgesetztes Substrat je mol Metall und Stunde beobachtet. Auch als Proteininhibitoren wurden Materialien untersucht, die durch katalytische Polymerisation in Wasser hergestellt wurden. Es hat sich ein vielseitiges Arbeitsgebiet herauskristallisiert, das Kolloid-, Polymer- und Koordinationschemie vereint.

Stichwörter: Homogene Katalyse • Kolloide • Polymerisationen • Polymerlatex - Wasserchemie

\section{Einleitung}

Die Durchführung katalytischer Polymerisationen in wässerigen Systemen (wässeriger katalytischer Polymerisationen) mag auf den ersten Blick als ein Widerspruch erscheinen. Schließlich ist die extreme Empfindlichkeit der in der technischen Olefinpolymerisation eingesetzten Ziegler- oder Phillips-Katalysatoren gegen Feuchtigkeit hinlänglich bekannt. Wasser bietet jedoch als Medium für Polymerisationsreaktionen eine Kombination von außergewöhnlichen Eigenschaften:

- Die hohe Wärmekapazität ermöglicht ein effektives Abführen der Polymerisationswärme.

- Die hohe Polarität führt zu einem völlig anderen Mischungsverhalten mit vielen Monomeren und Polymeren als bei organischen Lösungsmitteln.

[*] Dr. S. Mecking, Dr. A. Held, Dipl.-Chem. F. M. Bauers Institut für Makromolekulare Chemie und Freiburger Materialforschungszentrum der Albert-Ludwigs-Universität Freiburg Stefan-Meier-Straße 31, 79104 Freiburg (Deutschland) Fax: (+49) 761-203-6319

E-mail: stefan.mecking@makro.uni-freiburg.de
- Mit Tensiden können Dispersionen hydrophober Polymerpartikel in Wasser gegen Aggregation geschützt werden.

- Wasser ist unbrennbar und nicht toxisch.

Die vielseitigen aus diesem Eigenschaftsprofil resultierenden Möglichkeiten können an den etablierten nichtkatalytischen Polymerisationsverfahren veranschaulicht werden.

\subsection{Wässerige radikalische Polymerisation}

Die radikalisch initiierte Emulsionspolymerisation wird heute im großen Maßstab angewendet. ${ }^{[1-4]}$ Die Emulsionspolymerisation von Styrol und Butadien war in den 1940er Jahren parallel in den Vereinigten Staaten und in Deutschland entwickelt worden, um während der Kriegszeit einen Ersatz für natürlichen Kautschuk zu erhalten. Heute sind Vinylacetat, Acrylsäure und deren Ester, Butadien und Styrol die wichtigsten Monomere für die Emulsionspolymerisation.

Das Produkt von Emulsionspolymerisationen sind Polymerlatices. Ein Polymerlatex ist eine stabile Dispersion von Polymerpartikeln mit Größen von 50 bis $1000 \mathrm{~nm}$ in einem flüssigen Medium, meist Wasser. Latices werden auch als 
Polymerdispersionen bezeichnet. In solchen Latices müssen die Koagulation und das Ausfallen der Polymerpartikel verhindert werden. Dazu eignen sich die elektrostatische Stabilisierung (meist durch an der Partikeloberfläche adsorbierte niedermolekulare ionische Tenside) und die sterische Stabilisierung (zum Beispiel durch wasserlösliche Polymere, die adsorbiert oder kovalent an die Partikel gebunden werden). Die Mechanismen von Emulsionspolymerisationen sind komplex, und trotz intensiver Untersuchungen sind viele Details noch ungeklärt. Die radikalische und die katalytische Polymerisation unterscheiden sich zudem in den Mechanismen von Kettenwachstum, Ketteninitiierung und Kettentransfer oder -terminierung. Deshalb können die für die radikalische Polymerisation gewonnenen Einblicke nicht ohne weiteres auf die katalytische übertragen werden. Trotzdem kann eine stark vereinfachte Betrachtung hilfreich sein, um die zugrunde liegenden Prinzipien zu veranschaulichen.

Ein typisches Polymerisationsgemisch besteht zunächst aus Wasser, emulgiertem wasserunlöslichem MoEmulsionspolymerisation. nomer, Emulgator und wasserlöslichem Initiator. Ein wasserlösliches Radikal, z. B. aus $\mathrm{K}_{2} \mathrm{~S}_{2} \mathrm{O}_{8}$ gebildetes $\mathrm{SO}_{4}{ }^{--}$, wächst durch Anlagerung von Monomer, das in kleinen Mengen in der wässerigen Phase gelöst ist (Schema 1). Das resultierende wasserlösliche Oligoradikal wächst bis zu einer kritischen Größe, bei der es wasserunlöslich wird und auf sich selbst kollabiert („homogene Keimbildung“). Das resultierende Primärpartikel wird durch die Adsorption von Emulgator

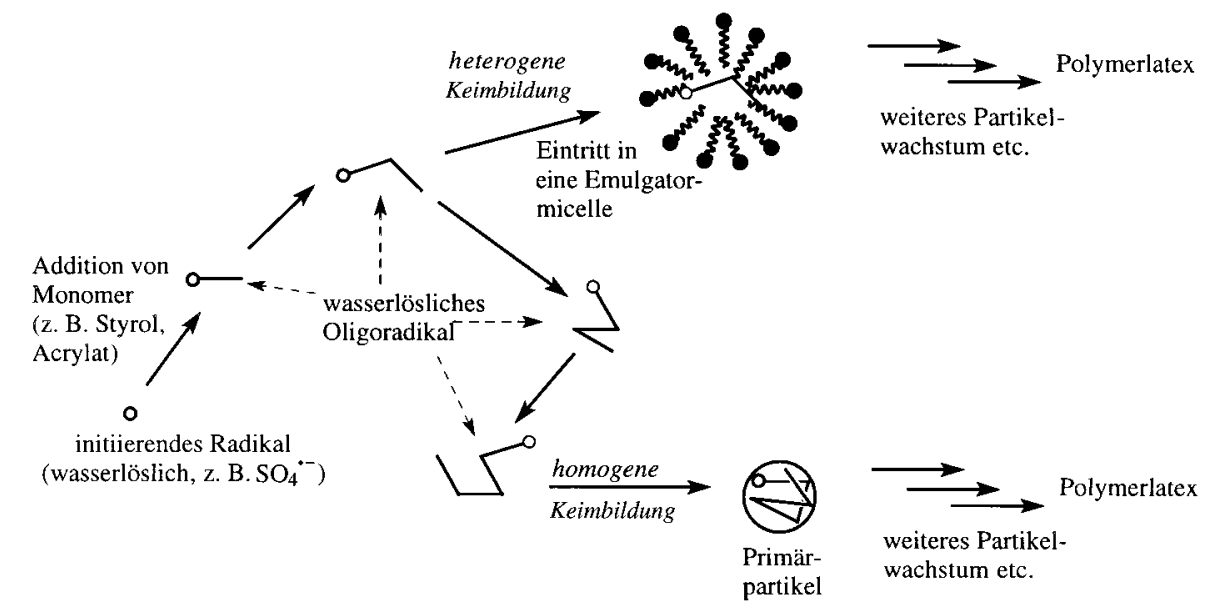

Schema 1. Vereinfachte Darstellung möglicher Partikelbildungs-Mechanismen in der radikalischen

Stefan Mecking, geboren im Oktober 1966, studierte Chemie an der RWTH Aachen. 1994 promovierte er im Arbeitskreis von W. Keim mit einer Arbeit über katalytische C-C-Verknüpfungen durch Palladiumkomplexe mit hemilabilen $\mathrm{Li}$ ganden. Nach einem 18-monatigen Postdoc-Aufenthalt als Feodor-LynenStipendiat in der Gruppe von $M$. Brookhart (University of North Carolina at Chapel Hill) trat er 1996 als Labor- und Projektleiter in die zentrale Forschung der Hoechst AG in Frankfurt ein. 1998 wechselte er an das Freiburger Materialforschungszentrum/Institut für Makromolekulare Chemie der Universität Freiburg, wo er sich derzeit habilitiert. Neben wässerigen katalytischen

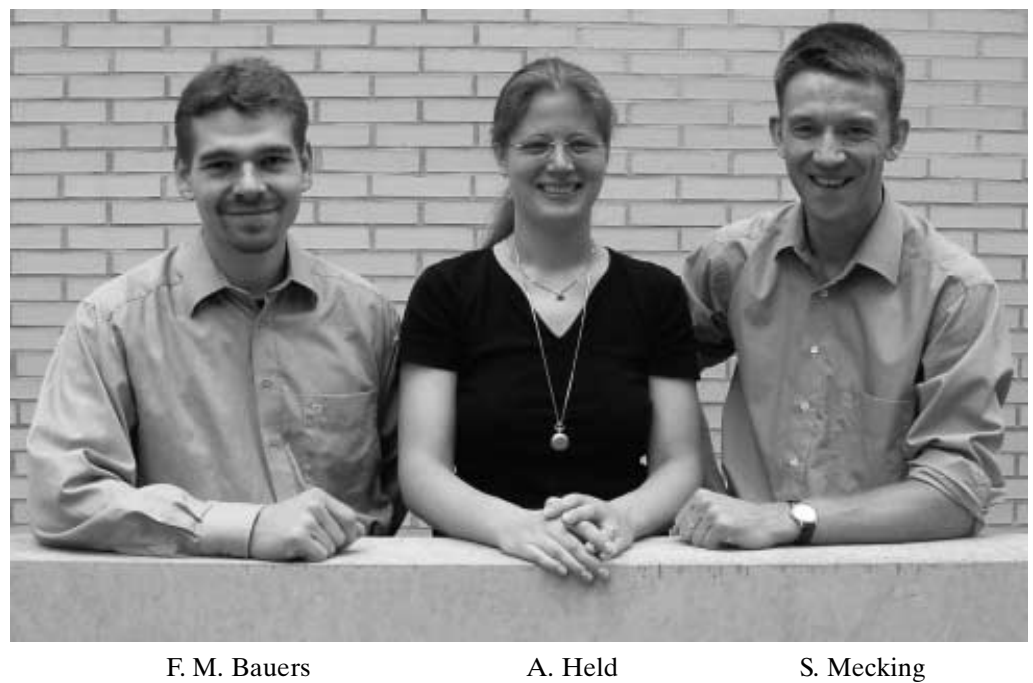

Polymerisationen interessieren ihn katalytisch aktive, kolloidale Polymer/Metall-Hybride. Kürzlich erhielt er für diese Arbeiten den Eugen-Graetz-Preis der Universität Freiburg.

Anke Held, geboren 1974 in Berlin, studierte Chemie in Marburg. Nach der Diplomarbeit unter Anleitung von W. Heitz wechselte sie 1998 nach Freiburg in die Gruppe von S. Mecking. Ihre Doktorarbeit zur wässerigen katalytischen Olefinpolymerisation durch Palladium(II)-Komplexe unter Anleitung von R. Mülhaupt hat sie kürzlich abgeschlossen und ist nun bei Basell in Ludwigshafen tätig.

Florian M. Bauers, geboren 1974 in Berlin, studierte Chemie in Freiburg. Anfang 2000 schloss er sein Studium mit einer Diplomarbeit über die Nickel(II)-katalysierte wässerige Ethylenpolymerisation ab. Seitdem führt er diese Arbeit in seiner Dissertation fort. Für herausragende Leistungen in Diplomarbeit und Studium erhielt er vor kurzem den Steinhofer-Preis. 
stabilisiert, und es erfolgt weiteres Partikelwachstum durch Polymerisation im Partikel. Alternativ kann das wasserlösliche Oligoradikal in eine Emulgatormicelle eindringen, bevor es seine kritische Kettenlänge erreicht, und so ein Partikel generieren (,heterogene Keimbildung“). Während der Emulsionspolymerisation dienen die emulgierten Monomertröpfchen als Reservoir für das Monomer, aber die Polymerisation findet üblicherweise nicht in diesen Tröpfchen statt, sondern in den Polymerpartikeln. Das Monomer diffundiert von den Monomertröpfchen durch die Wasserphase in die Partikel (Schema 2). (Eine andere Situation liegt bei der Miniemulsionspolymerisation vor, siehe Abschnitt 3.) Damit kann der Begriff Emulsionspolymerisation irreführend sein. In dieser Übersicht wird vielfach der Begriff „Polymerisation in Emulsion" verwendet, um eine katalytische Polymerisation zu beschreiben, die unter Emulsionsbedingungen durchgeführt wird. Damit sollen keinerlei Aussagen zum Mechanismus der Partikelbildung verbunden sein, da dieser meist nicht untersucht wurde. Außerdem soll keine Bildung stabiler Polymerlatices impliziert werden.

Die Viskosität der Reaktionsmischung erhöht sich während der Emulsionspolymerisation nur relativ wenig. Da die Viskosität eines wässerigen Latex wesentlich geringer ist als die einer Polymerlösung in einem organischen Lösungsmittel, können hohe Polymerausbeuten in einem vorgegebenen Reaktionsvolumen erzielt werden. Aus den erhaltenen Polymerlatices kann das Polymer durch Ausfällen isoliert werden. Für viele Anwendungen, z. B. in Farben und Lacken, werden die Latices jedoch direkt weiterverwendet. In solchen Anwendungen ist die Filmbildung ein Schlüsselschritt. Die Verdampfung des Dispersionsmediums führt zur Bildung eines Polymerfilms auf dem Substrat. Bei diesem Vorgang werden jährlich mehrere Millionen Tonnen Wasser verdampft. Die Unbrennbarkeit und die Umweltfreundlichkeit von Wasser sind dabei von besonderem Vorteil. Zusätzlich zu den existierenden großtechnischen Anwendungen in Farben, Lacken, Klebstoffen, der Papierherstellung und anderen Industriezweigen haben Polymerdispersionen ein interessantes Potential als Trägermaterialien im Mikromaßstab, z. B. für biomedizinische Anwendungen ${ }^{[5]}$ oder als Katalysatorträger. $^{[6-9]}$ Polymerlatices können auch als Substrate für polymeranaloge Reaktionen (d.h. Synthesen unter Verwendung eines Polymers als Substrat) eingesetzt werden. ${ }^{[10,11]}$ Aus all dem folgt, dass Polymerlatices nicht nur eine günstige Möglichkeit für Polymerproduzenten sind, „dem Kunden eine große Menge Wasser zu verkaufen“",12] sondern auch
Suspensionspolymerisation

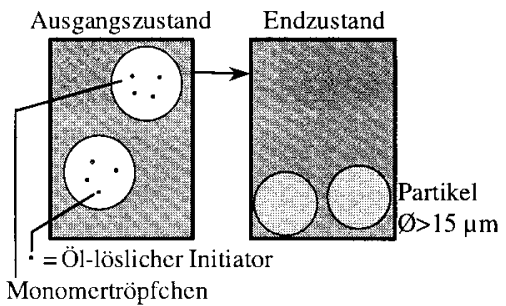

Emulsionspolymerisation

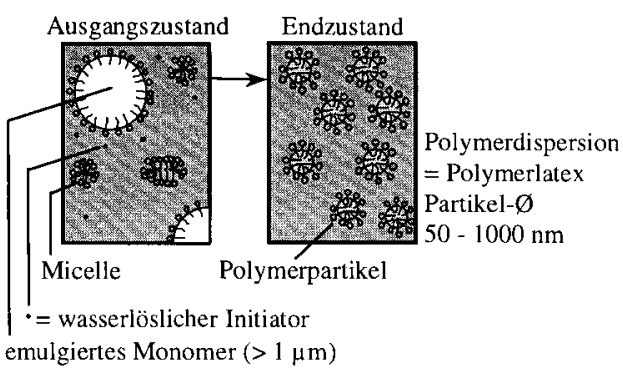

Schema 2. Schematische Darstellung der radikalischen Suspensions- und Emulsionspolymerisation. einzigartige Vorteile bezüglich der Anwendungen und des Polymerisationsverfahrens bieten.

Auch in der Suspensionspolymerisation wird ein wasserunlösliches olefinisches Monomer polymerisiert. Typischerweise wird eine Mischung aus Wasser, wasserunlöslichem Monomer, monomerlöslichem Initiator und möglicherweise einem Stabilisator heftig gerührt. Die Polymerisation erfolgt in den Monomertröpfchen (Schema 2). Die erhaltenen Partikel sind viel größer $(>15 \mu \mathrm{m})$ als bei der Emulsionspolymerisation und bilden keine stabilen Latices, sondern fallen während der Polymerisation aus. So können die in Ionenaustauscherharzen verwendeten Polystyrolperlen durch Suspensionspolymerisation hergestellt werden. ${ }^{[13]}$ Im Folgenden soll die Bezeichnung einer katalytischen Polymerisation als ,suspensionsartig“ lediglich ausdrücken, dass ein wasserunlösliches Monomer zu einem Polymer umgesetzt wird, welches sich während der Polymerisation absetzt, in der Regel in Abwesenheit eines Stabilisators.

In der Dispersionspolymerisation wird ein gelöstes Monomer zu einem unlöslichen Polymer polymerisiert, das in Form eines stabilen Latex anfällt. Mit Partikelgrößen von 1 bis $15 \mu \mathrm{m}$ kann die Dispersionspolymerisation den Partikelgrößenbereich zwischen Emulsions- und Suspensionspolymerisation abdecken.

Des Weiteren ist die wässerige Lösungspolymerisation gut bekannt, bei der ein wasserlösliches Monomer zu einem wasserlöslichen Polymer polymerisiert wird. ${ }^{[14]}$ Ein Beispiel ist die Herstellung von Polyacrylsäure im großen Maßstab durch radikalische Polymerisation. ${ }^{[15]}$ Wieder sind die Unbrennbarkeit und die Ungiftigkeit von Wasser sowie dessen hohe Wärmekapazität von Vorteil. In einigen Fällen ermöglicht die Polymerisation in homogener Lösung eine bessere Molekulargewichtssteuerung als die mehrphasige Polymerisation (siehe Abschnitt 6).

\section{2. Übergangsmetallkatalyse in wässerigen Medien}

Im Gegensatz zu der in Abschnitt 1.1 vorgestellten großtechnisch durchgeführten radikalischen Polymerisation wurden Übergangsmetall-katalysierte Koordinationspolymerisationen in Wasser bisher wenig untersucht. Der Hauptgrund dürfte sein, dass die in der kommerziellen katalytischen Polymerisation von Olefinen ${ }^{[16-19]}$ fast ausschließlich verwendeten Katalysatoren auf der Basis früher Übergangsmetalle extrem feuchtigkeitsempfindlich sind. Welche Faktoren bestimmen die Reaktivität und Stabilität eines gegebenen Katalysators gegen Wasser? Bei Metall-katalysierten Umsetzungen von olefinischen Substraten zu niedermolekularen Produkten oder zu Polymeren treten im Allgemeinen metallorganische Verbindungen, meist mit Metall-Alkyl-Bindungen, als aktive Spezies auf. Eine allgemeine Voraussetzung für katalytische Umsetzungen ist die Zugänglichkeit von Koordinationsstellen für die Anbindung des 
Substrats. Bei katalytischen Reaktionen in Wasser können unerwünschte Nebenreaktionen auftreten: Wasser kann Metall-Alkyl-Bindungen hydrolysieren, Koordinationsstellen blockieren oder das koordinierte olefinische Monomer oder andere Liganden angreifen. ${ }^{[20]}$ Bezüglich der ersten beiden vielleicht entscheidendsten - Punkte sind Komplexe später Übergangsmetalle wegen ihrer geringeren Oxophilie wesentlich weniger empfindlich als die früher Übergangsmetalle. Die Stabilität gegen Wasser wird durch die großtechnische kommerzielle Anwendung des Ruhrchemie-Rhone-Poulenc-Verfahrens eindrucksvoll belegt, ${ }^{[21]}$ bei dem Propylen mithilfe eines Rhodiumkatalysators in einem zweiphasigen wässerigen System hydroformyliert wird. Dies ermöglicht eine einfache Abtrennung des wasserlöslichen Katalysators von den apolaren Produkten. Eine Vielzahl niedermolekularer Verbindungen wurde schon durch metallorganische Katalyse in wässerigen Systemen hergestellt, und solche Reaktionen sind heutzutage keineswegs mehr exotisch. Im Vergleich dazu hat die katalytische Polymerisation in Wasser bisher wenig Aufmerksamkeit gefunden. In der umfassenden, von Cornils und Herrmann herausgegebenen Übersicht über die metallorganische Katalyse in wässerigen Systemen ${ }^{[21]}$ nimmt die Polymerisation nur einen kleinen Teil ein. ${ }^{[22]}$

Die Durchführung von katalytischen Polymerisationen in Wasser ist jedoch ein attraktives Ziel. Die Betrachtungen zu radikalischen wässerigen Polymerisationen in Abschnitt 1.1 veranschaulichen die einzigartigen Vorteile von Wasser als Reaktionsmedium. Katalytische Polymerisationen ermöglichen den Zugang zu vielen Polymermikrostrukturen, die durch radikalische Polymerisation nicht hergestellt werden können. In den letzten Jahren wurden beachtliche Fortschritte auf dem Gebiet der wässerigen katalytischen Polymerisation erzielt. Dieser Trend mag mit Fortschritten bei der Olefinpolymerisation durch Komplexe später Übergangsmetalle im Allgemeinen oder einem steigenden Bedarf an umweltfreundlichen Produkten und Verfahren zusammenhängen und vielleicht auch einfach mit der Faszination durch dieses Thema und die Möglichkeiten, die aus der Kombination von Polymer-, Organometall- und Kolloidchemie resultieren. Interessanterweise haben auch andere nichtradikalische Polymerisationen in wässerigen Emulsionen, wie die Polykondensation $^{[23]}$ und die ionische Polymerisation, ${ }^{[24-27]}$ in jüngerer Zeit vermehrt Aufmerksamkeit gefunden. Auch Metall-vermittelte Reaktionen wie die Polymerisation über SuzukiKupplung in wässerigen Medien, ${ }^{[28,29]}$ wässerige ATRP (Atom Transfer Radical Polymerization $)^{[30-32]}$ und die Polymerisation von Acetylen ${ }^{[33,34]}$ wurden kürzlich untersucht. In dieser Übersicht werden wässerige katalytische Polymerisationen von olefinischen Monomeren behandelt. Um die besonderen Eigenschaften von Wasser nutzen zu können, ist es erforderlich, dass dieses als ein Hauptbestandteil im Reaktionsmedium vorliegt. Katalytische Polymerisationen, die in organischen Lösungsmitteln in Gegenwart eines kleinen Wasseranteils durchgeführt wurden, werden daher im Folgenden nicht betrachtet. Beim Vergleich der angegebenen Katalysatoraktivitäten ist zu beachten, dass sie unter stark variierenden Bedingungen von verschiedenen Autoren bestimmt wurden. Deshalb sollten die Daten lediglich als ein Maß für die Größenordnung der Aktivität verstanden werden. Dassel- be gilt für die Molekulargewichte der Polymere. Um einen Vergleich der Polymerisationen unterschiedlicher Monomere (mit unterschiedlichen Molekulargewichten) durch Katalysatoren mit unterschiedlichen Metallzentren zu ermöglichen, sind die Aktivitäten als Zahl katalytischer Umsetzungen pro Stunde angegeben $\left(\mathrm{TO} \mathrm{h}^{-1} ; \mathrm{TO}=\right.$ mol umgesetztes Substrat je mol Metall).

\section{Copolymerisation von Kohlenmonoxid mit Olefinen}

Die katalytische Copolymerisation von Olefinen mit Kohlenmonoxid hat wegen der Verwendung von kostengünstigen Ausgangsstoffen und der Materialeigenschaften der Copolymere breites Interesse gefunden. ${ }^{[35-37]}$ Bei dieser Polymerisation werden Kohlenmonoxid und Olefin streng alternierend eingebaut [Gl.(1)]. Ein alternierendes Ethylen-Kohlen-

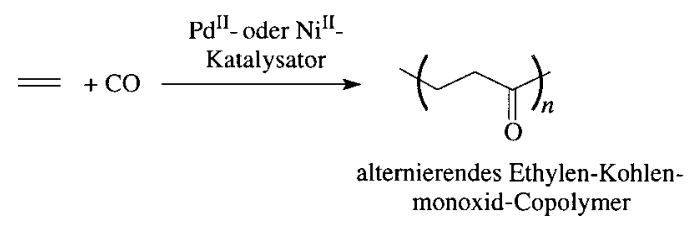

monoxid-Copolymer schmilzt bei $257^{\circ} \mathrm{C}$. Der Einbau von Propylen erniedrigt den Schmelzpunkt, wodurch die Verarbeitung der Materialien möglich wird (z.B. 6 Mol-\% Propylen: $\left.T_{\mathrm{m}}=220^{\circ} \mathrm{C}\right) .{ }^{[43]}$ Diese teilkristallinen Ethylen-Propylen-Kohlenmonoxid-Terpolymere werden seit 1995 von Shell als neue technische Thermoplaste unter dem Markennamen Carilon kommerzialisiert, und BP hat ähnliche Materialien mit der Bezeichnung Ketonex entwickelt. ${ }^{[38,39]}$ Kürzlich wurde allerdings von Shell angekündigt, dieses Geschäft aufgeben zu wollen. ${ }^{[40]}$ Die Polyketone zeichnen sich durch eine hohe Widerstandsfähigkeit gegen unpolare organische Lösungsmittel und eine gute Abriebfestigkeit aus. ${ }^{[41]}$ Die große Zahl an Carbonylgruppen macht sie jedoch empfindlich gegen UV-Strahlung; zudem können bei den für die Verarbeitung erforderlichen hohen Temperaturen Kondensationsreaktionen zu unerwünschter Quervernetzung führen. Diese Materialien sind bemerkenswerterweise die einzigen auf Olefinen basierenden kommerziell verfügbaren Polymere, die eine große Zahl an sauerstoffhaltigen polaren Einheiten enthalten und durch katalytische Polymerisation hergestellt werden.

Hinsichtlich des Katalysatorsystems war die Entdeckung, dass kationische Palladium(II)-Komplexe mit chelatisierenden Liganden wesentlich höhere Aktivitäten in der EthylenKohlenmonoxid-Copolymerisation aufweisen als die bis dahin bekannten Systeme, ein Durchbruch. ${ }^{[42,43]}$ Zusätzlich zu Diphosphanen wurde eine Vielzahl an Liganden mit Stickstoff- ${ }^{[4,45]}$ und anderen Donoratomen ${ }^{[46]}$ gefunden, die für die Olefin-Kohlenmonoxid-Copolymerisation geeignet sind. Als Reaktionsmedium wird meist Methanol verwendet, was die Stabilität dieser Katalysatoren in protischen Medien aufzeigt. $^{[47]}$ 
Die erste Untersuchung einer katalytischen Olefin-Kohlenmonoxid-Copolymerisation wurde in Wasser als Reaktionsmedium durchgeführt! In einem 1948 angemeldeten Patent beschreiben Reppe und Magin die Reaktion von Ethylen mit Kohlenmonoxid in Gegenwart einer wässerigen Lösung von Kaliumtetracyanonickelat(II) bei $150^{\circ} \mathrm{C}$ und 150 bar. ${ }^{[48]}$ Neben den Oligomeren $\mathrm{H}\left[\mathrm{CH}_{2} \mathrm{CH}_{2} \mathrm{C}(=\mathrm{O})\right]_{n} \mathrm{C}_{2} \mathrm{H}_{5}$ und $\mathrm{H}\left[\mathrm{CH}_{2} \mathrm{CH}_{2} \mathrm{C}(=\mathrm{O})\right]_{n} \mathrm{OH}(n=1,2)$ wurden höhermolekulare, feste „Polyketone“ erhalten. Diese Reaktion kann als sehr frühes Beispiel für eine wässerige katalytische Olefin,,polymerisation “ gesehen werden. ${ }^{[49]}$ In jüngerer Zeit sind mehrere Arbeiten zu Ethylen-Kohlenmonoxid-Copolymerisationen in Wasser als Reaktionsmedium erschienen, bei denen kationische Palladium(II)-Komplexe mit wasserlöslichen zweizähnigen Liganden eingesetzt wurden (zur Löslichkeit der Monomere in Wasser siehe Lit. [50]). Mit den sulfonierten Stickstoff- und Phosphanliganden 1 bzw. 2 beobachteten Sen et al. in der Ethylen-Kohlenmonoxid-Copolymerisation moderate

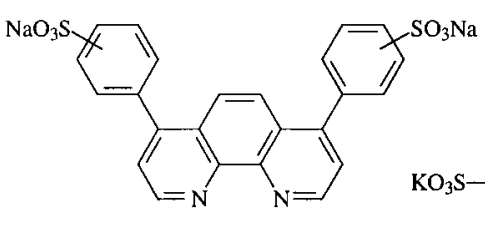

1

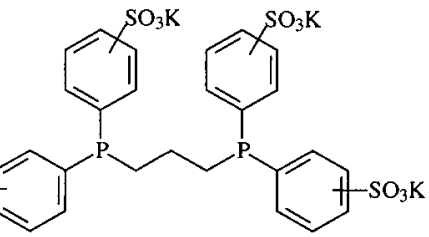

2
Aktivitäten bis ca. $10^{2} \mathrm{TOh}^{-1}{ }^{\left[{ }^{[51]}\right.}$ Ihre Reaktionsbedingungen waren milder als die von Reppe und Magin: Die Polymerisation wurde bei $50^{\circ} \mathrm{C}$ und jeweils 35 bar Ethylen- und Kohlenmonoxiddruck durchgeführt (bei einer Reaktionszeit von 22 Stunden mit einem in situ aus $\left[\mathrm{Pd}^{\mathrm{II}}\left(\mathrm{NCCH}_{3}\right)_{4}\right]\left(\mathrm{BF}_{4}\right)_{2}$ und dem zweizähnigen Liganden hergestellten Katalysator). Außerdem wurde ein alternierendes Propylen-KohlenmonoxidCopolymer mit einem Molekulargewicht von $1.4 \times 10^{4} \mathrm{~g} \mathrm{~mol}^{-1}$ (gegen Polystyrolstandards) hergestellt, jedoch mit niedrigeren Aktivitäten.

Bei der Untersuchung der wässerigen Ethylen-Kohlenmonoxid-Copolymerisation mit einem sehr ähnlichen Katalysatorsystem, mit demselben Liganden 2, haben Sheldon et al. über signifikant höhere Aktivitäten bis $1.5 \times 10^{4} \mathrm{TOh}^{-1}$ berichtet. ${ }^{[52]}$ Diese höheren Aktivitäten, die denen ähneln, die mit 1,3-Bis(diphenylphosphanyl)propan (dppp) als nichtsulfoniertem „Vorbild“ für $\mathbf{2}$ in nichtwässerigen Polymerisationen in Methanol erzielt werden, wurden der höheren Reinheit des sulfonierten Phosphanliganden sowie dem $\mathrm{Zu}$ satz von Brønstedt-Säure, die die Katalysatorstabilität erhöht, zugeschrieben. Die Molekulargewichte der Copolymere ähneln mit $M_{\mathrm{w}} \leq 5 \times 10^{4} \mathrm{~g} \mathrm{~mol}^{-1}$ und $M_{\mathrm{w}} / M_{\mathrm{n}} \approx 2$ denen der Materialien, die in Methanol unter sonst vergleichbaren Bedingungen erhalten werden. Die konstante Produktivität über mehrere Stunden zeugt von einer hohen Katalysatorstabilität in der wässerigen Reaktion. ${ }^{[52,53]}$ Änderungen der Länge des „Ligandenrückgrates“ $\left(\mathrm{Ar}_{2} \mathrm{P}\left(\mathrm{CH}_{2}\right)_{n} \mathrm{PAr}_{2}\right.$ mit $n=$ 2-5) ergaben, dass die besten Ergebnisse in Bezug auf Aktivität und Molekulargewicht mit $n=3$ (2) erzielt werden. ${ }^{[53]}$ Dieser Trend ist ähnlich wie in nichtwässerigen Polymerisationen in Methanol, d.h., abgesehen von der
Löslichkeit werden offensichtlich keine für die wässerige Polymerisation spezifischen Anforderungen an die Ligandenstruktur in diesem bestimmten Katalysatorsystem gestellt. Speziell entworfene wasserlösliche Liganden mit zwei sulfonierten Einheiten an einem $\mathrm{C}_{3}$-Ligandenrückgrat $(3)^{[54]}$ oder hydroxylierte neutrale Liganden wie $\mathbf{5}^{[55]}$ liefern aktivere Katalysatoren als 2.
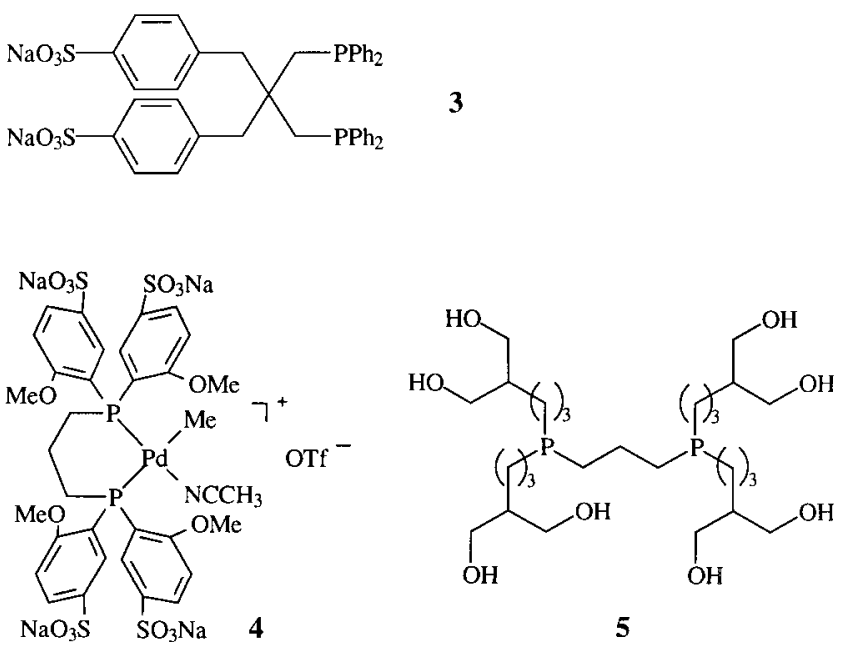

5

Kationische Palladiumkatalysatoren, die an jeweils einer ortho-Position der Arenringe von Diphosphanliganden wie 2 Methoxy-substituiert sind, zeigen deutlich höhere Katalysatorleistungen. ${ }^{[47]}$ Mit dem vollständig $o$-OMe-substituierten Analogon von Ligand 2 werden in der wässerigen Polymerisation Ethylen-Kohlenmonoxid-Copolymere mit $M_{\mathrm{w}} \leq 1.2 \times$ $10^{5} \mathrm{~g} \mathrm{~mol}^{-1}$ und engen Polydispersitäten $\left(M_{\mathrm{w}} / M_{\mathrm{n}} \approx 2\right)$ erhalten. Beim Einsatz des Komplexes 4 dieses Liganden werden hohe Aktivitäten von $6.1 \times 10^{4} \mathrm{TOh}^{-1}\left(90^{\circ} \mathrm{C}, 60\right.$ bar Ethylen/CO $1: 1,1$ h Reaktionszeit) genannt. ${ }^{[56]}$

Wie schon erwähnt, können die Eigenschaften der EthylenKohlenmonoxid-Copolymere durch die Einführung weiterer Olefine als Termonomere variiert werden. Außerdem sind 1-Olefin-Kohlenmonoxid-Copolymere gut bekannt. Die Eigenschaften dieser Copolymere hängen stark von ihrer Zusammensetzung ab. So sinkt die Glastemperatur von 1-Olefin-Kohlenmonoxid-Copolymeren mit steigender Kettenlänge des 1-Olefins (Propylen bis 1-Dodecen) von Raumtemperatur auf fast $-60^{\circ} \mathrm{C}$ ab. ${ }^{[37]} \mathrm{Im}$ Gegensatz zu den polaren Ethylen-Kohlenmonoxid-Copolymeren sind Copolymere mit längerkettigen 1-Olefinen hydrophob. Ethylen-1Olefin-Kohlenmonoxid-Terpolymere und 1-Olefin-Kohlenmonoxid-Copolymere können in wässeriger Polymerisation hergestellt werden. ${ }^{[51,54,55]}$ Für die 1-Olefin-Copolymerisation sind Katalysatoren mit vollständig Alkyl-substituierten Diphosphanen wie 5 (im Vergleich zu den Phenylsubstituenten in dppp) besonders geeignet.

In den genannten Copolymerisationen fallen die Polyketone während der Reaktion als Feststoff aus. ${ }^{[33-56]}$ Jedoch können in Gegenwart von Emulgatoren wie Natriumdodecylsulfat (SDS) bei geeignet gewählten Bedingungen stabile Dispersionen erhalten werden. Solche Polymerisationen können mit wasserlöslichen Katalysatoren mit Liganden des Typs 5 durchgeführt werden. ${ }^{[57]}$ Bei Einsatz der Miniemulsions- 
technik (siehe Abschnitt 3) können auch wasserunlösliche Katalysatoren verwendet werden, um stabile Latices herzustellen. ${ }^{[58]}$ Damit kann der Aufwand für die Ligandensynthese verringert werden. Es wurden stabile Latices von EthylenUndec-10-ensäure-Kohlenmonoxid-Terpolymeren $\left(M_{\mathrm{w}}=7 \times\right.$ $10^{4} \mathrm{~g} \mathrm{~mol}^{-1}$ gegen PMMA-Standards) und von 1-Olefin-Kohlenmonoxid-Copolymeren $\left(M_{\mathrm{w}}=2 \times 10^{4} \mathrm{~g} \mathrm{~mol}^{-1}\right)$ erhalten. 1-Olefin-Kohlenmonoxid-Copolymere sind für die Filmbildung gut geeignet, da ihr Glasübergang unterhalb von Raumtemperatur erfolgt. Außerdem können Latices mit hohem Feststoffgehalt hergestellt werden.

Das Kettenwachstum bei der wässerigen Ethylen-Kohlenmonoxid-Copolymerisation durch alternierende Insertion der Monomere in eine Palladium-Acyl- bzw. Palladium-AlkylSpezies ist identisch mit dem bei der gut untersuchten Copolymerisation in Methanol. Ein bedeutender mechanistischer Unterschied ist aber die Hydrolyse als ein Kettenübertragungsschritt: In nichtwässerigen Polymerisationen führt die Methanolyse eines Palladium-Alkyl-Komplexes zu einer Pd-OMe-Spezies, die weiteres Kettenwachstum initiiert und damit eine neue $\mathrm{CH}_{2} \mathrm{CH}_{2} \mathrm{C}(=\mathrm{O}) \mathrm{OMe}$-Endgruppe im Polymer erzeugt. Im Gegensatz dazu liefert die Hydrolyse eine $\mathrm{Pd}-\mathrm{OH}-\mathrm{Spezies}$, die mit Kohlenmonoxid $\mathrm{zu}$ einem Palladiumhydrid reagiert, bevor die nächste Kette initiiert wird (Schema 3). Daher werden in wässerigen Reaktionen bevorzugt oder ausschließlich Polyketone mit zwei KetoEndgruppen erhalten. ${ }^{[52,54,59]}$ Die Reaktion von Pd-OHSpezies mit Kohlenmonoxid kann als „Reaktivierungsweg“ nach der Hydrolyse einer Metall-Alkyl-Bindung betrachtet werden, der für wässerige Reaktionen in Gegenwart von Kohlenmonoxid spezifisch ist.

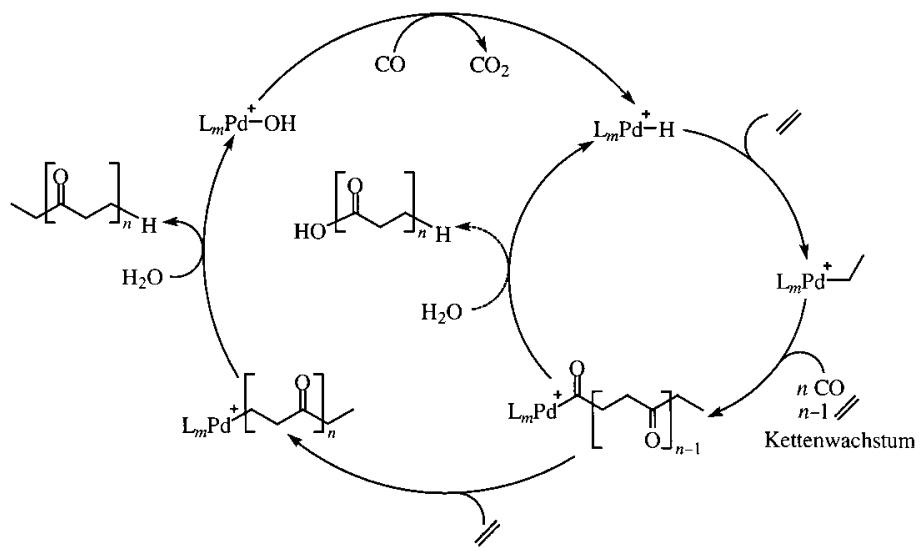

Schema 3. Mechanismus der wässerigen Ethylen-Kohlenmonoxid-Copolymerisation.

Aus den vorhandenen Daten kann geschlossen werden, dass die wässerige Ethylen-Kohlenmonoxid-Copolymerisation prinzipiell mit ähnlichen Aktivitäten wie der in Methanol durchgeführte kommerzielle Prozess ablaufen kann. Dabei können hochmolekulare Polyketone erhalten werden. Offensichtliche Vorteile von Wasser als Reaktionsmedium für solche Polymerisationen sind dessen Unbrennbarkeit und Umweltfreundlichkeit. Die Zugänglichkeit von stabilen Latices kann möglicherweise neue Anwendungen eröffnen, die zudem keine Verarbeitung bei nachteilig hohen Temperaturen erfordern.

\section{Polymerisation von Ethylen und 1-Olefinen}

In der kommerziellen Herstellung von Polyolefinen werden Ziegler- oder Phillips-Katalysatoren eingesetzt, die auf frühen Übergangsmetallen (Ti, Zr, Cr, V) basieren. ${ }^{[16-19]}$ Wegen ihrer hohen Oxophilie erfordern solche Katalysatoren streng wasserfreie Bedingungen. In den letzten Jahren hat die Entdeckung mehrerer neuer Typen von Katalysatoren für die Ethylen- und 1-Olefinpolymerisation auf der Basis von Komplexen später Übergangsmetalle ein starkes Interesse an diesem Gebiet hervorgerufen. ${ }^{[18,60-62]}$ Wegen der geringeren Oxophilie sind solche Katalysatoren in polaren Medien wesentlich stabiler.

Durch radikalische Emulsionspolymerisation werden Latices von Polyethylen niedriger Dichte (LDPE) als Spezialprodukt hergestellt. ${ }^{[63]} \mathrm{Zu}$ den Nachteilen dieses Prozesses zählen die Notwendigkeit, bei sehr hohem Druck (2000 bar) zu arbeiten, und die geringe Variabilität bezüglich der Einstellung der Polymermikrostruktur.

Über eine Ethylenpolymerisation ${ }^{[50]}$ in Wasser mit dem Rhodiumkomplex $\left[(\mathrm{N} \frown \mathrm{N} \frown \mathrm{N}) \mathrm{RhMe}\left(\mathrm{OH}_{2}\right)(\mathrm{OH})\right]^{+}$berichteten Flood und Mitarbeiter $1993(\mathrm{~N} \frown \mathrm{N} \frown \mathrm{N}=1,4,7-$ Trimethyl-1,4,7-triazacyclononan; in dieser Übersicht bezeichnet $\mathrm{X} \frown \mathrm{Y}$ einen zweizähnigen und $\mathrm{X} \frown \mathrm{Y} \frown \mathrm{Z}$ einen dreizähnigen Liganden, der über die Atome $\mathrm{X}$ und $\mathrm{Y}$ bzw. $\mathrm{X}, \mathrm{Y}$ und $\mathrm{Z}$ koordiniert). ${ }^{[64]}$ Ein niedermolekulares Polyethylen $\left(M_{\mathrm{w}}=\right.$ $5 \times 10^{3} \mathrm{~g} \mathrm{~mol}^{-1}$ ) wurde bei Raumtemperatur und 60 bar Ethylendruck nach einer Reaktionszeit von 90 Tagen erhalten. Obgleich die Reaktivität mit 1 TO pro Tag sehr gering war, war diese Umsetzung ein wichtiger Präzedenzfall für die wässerige katalytische Ethylenpolymerisation. Bei höheren Temperaturen konnte die Hydrolyse der Rh-Alkyl-Bindung als unerwünschte Konkurrenzreaktion zum Kettenwachstum beobachtet werden.

1995 berichteten Brookhart und Mitarbeiter, dass kationische, Diimin-substituierte Palladiumkomplexe des Typs 6 Ethylen in organischen Lösungsmitteln wie Dichlormethan zu

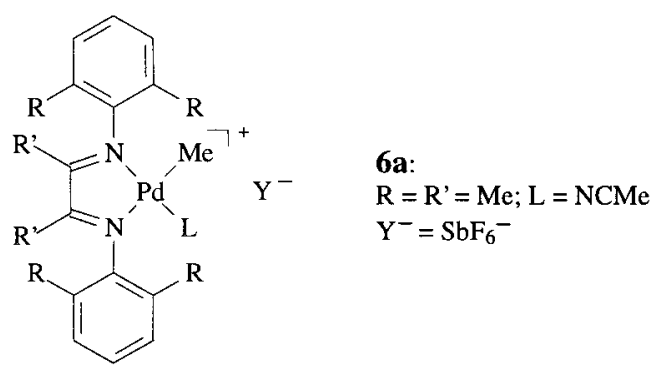

6

hochmolekularen, hochverzweigten Materialien polymerisieren können. Die besondere Struktur der Ethylenhomopolymere ist auf die Eigenschaft des Metallzentrums zurückzuführen, zwischen den Insertionen an der wachsenden Polymerkette „entlang zu laufen““[65, 66] In einem umfangreichen Patent zu diesen Polymerisationsreaktionen zeigten McLain et al. an einigen Beispielen, dass diese auch in Wasser durchgeführt werden können. ${ }^{[67]}$ Nach detaillierten Unter- 
suchungen in unserer Gruppe ist der Katalysator in dieser suspensionsartigen wässerigen Polymerisation bemerkenswert stabil::[68,73] Über einen Zeitraum von 16 Stunden oder länger wird Ethylen mit konstanter Aktivität polymerisiert; bei einem leicht erhöhten Ethylendruck von 20 bar liefert $\mathbf{6 a}$ als Katalysator mit $900 \mathrm{TOh}^{-1}$ ähnliche Aktivitäten wie in nichtwässerigen Polymerisationen in Dichlormethan. Diese hohe Stabilität ist jedoch auf eine „Verkapselung“ des wasserunlöslichen Katalysators im gebildeten hydrophoben Polymer zurückzuführen, was den Katalysator gegen einen Kontakt mit Wasser schützt. In Einklang damit sind wässerige Lösungen von wasserlöslichen Katalysatoren mit Sulfonatsubstituierten Diiminliganden bei der Ethylenpolymerisation inaktiv ${ }^{\left[{ }^{[9]}\right.}$ Mechanistische Studien ergaben, dass ein Komplex des Typs 6 in einer wasserhaltigen Lösung stabil ist, d.h., dass weder die Pd-Me-Gruppe noch der Diiminligand hydrolysiert wird. Bei Zusatz von Ethylen tritt jedoch sofortige Zersetzung ein. ${ }^{[68]} \mathrm{Im}$ Zusammenhang mit wässerigen Polymerisationen ist es zudem von Interesse, ob Wasser mit dem Monomer um die Koordination am Metallzentrum konkurrieren und so für die Polymerisation benötigte Koordinationsstellen blockieren kann. Die Zugabe von Ethylen zum Wasserkomplex $\left[\left(\mathrm{N}^{\frown} \mathrm{N}\right) \mathrm{PdMe}\left(\mathrm{OH}_{2}\right)\right]^{+}$bei $-40^{\circ} \mathrm{C}$ führte zur sauberen $\mathrm{Um}$ setzung zum bekannten ${ }^{[6]}$ Ethylenkomplex $\left[\left(\mathrm{N}^{\frown} \mathrm{N}\right) \mathrm{PdMe}-\right.$ $\left.\left(\mathrm{H}_{2} \mathrm{C}=\mathrm{CH}_{2}\right)\right]^{+} \cdot{ }^{\left[70,{ }^{73]}\right.}$ Dies zeigt, dass Wasser keine signifikante Neigung zur Blockierung der Koordinationsstelle in diesem kationischen Palladiumsystem hat.

Während bei der traditionellen Polymerisation in Dichlormethan unter ähnlichen Reaktionsbedingungen (Temperatur, Ethylendruck) hochviskose, flüssige Polyethylene $\left(M_{\mathrm{w}}=3 \times\right.$ $10^{4} \mathrm{~g} \mathrm{~mol}^{-1}$, ca. 100 Verzweigungen pro 1000 Kohlenstoffatome, $T_{\mathrm{g}}=-70^{\circ} \mathrm{C}$ ) erhalten werden, führt die Polymerisation mit 6a in wässeriger Suspension zu gummiartigen, amorphen Feststoffen $\left(M_{\mathrm{w}}=2 \times 10^{5} \mathrm{~g} \mathrm{~mol}^{-1}, M_{\mathrm{w}} / M_{\mathrm{n}}=2-3\right.$, ca. 70 Verzweigungen pro 1000 Kohlenstoffatome, $T_{\mathrm{g}}=-45^{\circ} \mathrm{C}$ ). Dieser Effekt ist nicht auf eine denkbare direkte Wechselwirkung von Wasser mit dem Katalysator, sondern eher auf die unterschiedliche lokale Umgebung der katalytisch aktiven Zentren während der Polymerisation zurückzuführen (heterogene wässerige Suspension des im Polymer verkapselten Katalysators statt homogener Lösung). ${ }^{[68]}$ Es sei darauf hingewiesen, dass die Ethylenpolymerisation mit $\mathbf{6}$ als katalytische wässerige Synthese eines hyperverzweigten Polyolefins betrachtet werden kann. ${ }^{[70,71]}$ Es wurde beansprucht, dass durch die Polymerisation von 1-Olefinen mit Katalysatoren des Typs $\mathbf{6}$ in wässeriger Emulsion stabile Latices erhalten werden. ${ }^{[72]}$

Für viele potentielle Anwendungen wäre die Synthese von weitgehend linearem Polyethylen mit definiertem Kristallinitätsgrad in wässeriger Emulsion von Interesse. Vor kurzem haben wir und Spitz et al. unabhängig über eine Nickel(II)katalysierte Polymerisation von Ethylen zu linearem Material in wässeriger Emulsion berichtet. ${ }^{[73,74]}$ Die neutralen Nickel(II)-Komplexe 7 und 8 (Schema 4) mit bekannten $\mathrm{P} \frown \mathrm{O}$ Liganden $^{[75-78]}$ erwiesen sich als geeignete Katalysatorvorläufer. Mit dem wasserlöslichen Katalysatorvorläufer $\mathbf{7 a}$ konnten stabile Latices von niedermolekularem Polyethylen erhalten werden: ${ }^{[73,79,80]}$ beispielsweise eine Polyethylendispersion mit $M_{\mathrm{w}}=3 \times 10^{3} \mathrm{~g} \mathrm{~mol}^{-1}$ und $M_{\mathrm{w}} / M_{\mathrm{n}}=2-3 \mathrm{mit}$

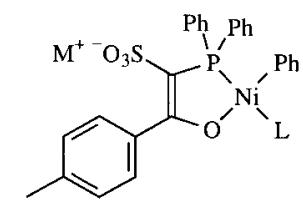

7

$\mathrm{L}=\mathrm{PPh}_{3}$ oder Pyridin

7b: $\mathrm{M}^{+}=\mathrm{H}_{33} \mathrm{C}_{16} \mathrm{NMe}_{3}^{+}$(lipophil) 7a: $\mathrm{M}^{+}=\mathrm{Na}^{+}$(hydrophil)

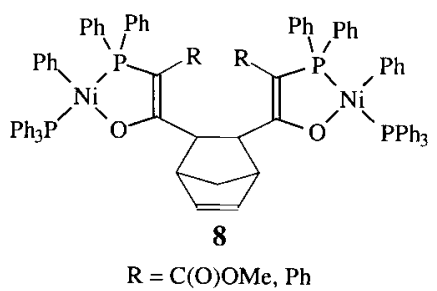

(Aktivierung von $\mathrm{PPh}_{3}$-Komplexen des Typs 7 oder 8 zur Polymerisation durch Phosphanfänger)
Schema 4. Neutrale Nickel(II)-Komplexe für die Ethylenpolymerisation in wässeriger Emulsion.

$10^{3} \mathrm{TOh}^{-1}\left(70^{\circ} \mathrm{C}, 50\right.$ bar Ethylendruck). Die Katalysatoren sind in der wässerigen Polymerisation über Stunden stabil. Mit 8 wurden in wässeriger Emulsion wesentlich höhere Aktivitäten bis $3 \times 10^{4} \mathrm{TOh}^{-1}$ beobachtet, jedoch waren die mit diesen lipophilen Katalysatorvorläufern hergestellten Latices nicht stabil. ${ }^{[74,81]}$ Die Katalysatoraktivitäten und die Polymer-Molekulargewichte sind bei der wässerigen Polymerisation geringer als bei der traditionellen Polymerisation in nichtwässerigen organischen Medien wie Toluol. Dies kann zu einem großen Teil auf eine geringere Kettenwachstumsgeschwindigkeit als Folge einer begrenzten lokalen Ethylenkonzentration am katalytisch aktiven Zentrum zurückgeführt werden. ${ }^{[79]}$ Daraus lässt sich schließen, dass zur Erhöhung der Polymerisationsgeschwindigkeit unter anderem gute Katalysatoraktivitäten bei relativ niedrigen Ethylenkonzentrationen von Vorteil sein sollten. ${ }^{[82]}$

Durch die Einführung von elektronenziehenden perfluorierten Substituenten am zweizähnigen $\mathrm{P} \frown$ O-Liganden (9, Schema 4) haben Claverie et al. hochaktive Katalysatoren erhalten. ${ }^{[83,84]}$ Mit beeindruckenden Aktivitäten bis $1.7 \times$ $10^{5} \mathrm{TOh}^{-1}$ wurde niedermolekulares, lineares Material $\left(M_{\mathrm{w}}=3 \times 10^{3} \mathrm{~g} \mathrm{~mol}^{-1}\right)$ in wässeriger Emulsion bei einem Ethylendruck von 25 bar hergestellt.

Hochmolekulares Polyethylen wurde in wässerigen Polymerisationen mit einer anderen Klasse von Katalysatoren erhalten. ${ }^{[79,88]}$ In der traditionellen Polymerisation in Toluol entstehen mit geeigneten $\mathrm{P} \frown \mathrm{O}$-substituierten Katalysatoren und mit den kürzlich entdeckten Salicylaldimin-substituierten Katalysatoren Polymere mit ähnlich hohen maximalen $M_{\mathrm{w}}{ }^{-}$ Werten. ${ }^{[62,76,77,85-87]} \mathrm{Im}$ Gegensatz zu den zuvor diskutierten $\mathrm{P} \frown \mathrm{O}$-substituierten Katalysatoren lieferte der letztere Typ von Komplexen auch in wässerigen Systemen hochmolekulares Material. Teilkristalline Polyethylene mit einem $M_{\mathrm{n}}$-Wert bis $10^{5} \mathrm{~g} \mathrm{~mol}^{-1}$ bei relativ engen Polydispersitäten $\left(M_{\mathrm{w}} / M_{\mathrm{n}}=2-4\right)$ sind in suspensionsartigen Polymerisationen mit den Katalysatorvorläufern 10 zugänglich. Obgleich diese Katalysatoren nur begrenzt gegen Wasser stabil sind, werden bei Raumtemperatur Aktivitäten von $3 \times 10^{3} \mathrm{TOh}^{-1}$ beobachtet. Wie die mit diesen Katalysatoren in traditionellen 


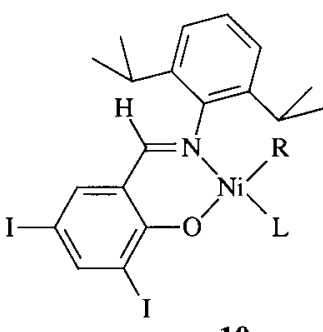

10
$\mathrm{R}=\mathrm{Ph}, \mathrm{L}=\mathrm{PPh}_{3}$

$\mathrm{R}=\mathrm{Me}, \mathrm{L}=$ Pyridin

(Aktivierung der $\mathrm{PPh}_{3}$-Komplexe für die Polymerisation durch $\left[\mathrm{Rh}\left(\mathrm{H}_{2} \mathrm{C}=\mathrm{CH}_{2}\right)_{2}(\mathrm{acac})\right]$ als Phosphanfänger)

Polymerisationen in organischen Lösungsmitteln erhaltenen Polymere weisen auch die Polyethylene aus der wässerigen Polymerisation eine moderate Zahl an Methylverzweigungen auf. Insgesamt hat die Gegenwart von Wasser, wie auch im Fall der mit 7-9 erhaltenen linearen Polyethylene, keinen Einfluss auf die grundlegende Polymermikrostruktur. Die Polymerkristallinität kann durch die Verwendung von Norbornen als Comonomer beeinflusst werden. Hochmolekulare, amorphe Ethylen-Norbornen-Copolymere, die bei Raumtemperatur verfilmen, ließen sich in wässerigen Polymerisationen herstellen. ${ }^{[79]}$

Die Synthese von stabilen Latices erfordert die Bildung und Stabilisierung von Primärpartikeln. In der klassischen radikalischen Emulsionspolymerisation werden wasserlösliche Initiatoren verwendet. Das Kettenwachstum liefert zunächst wasserlösliche Oligoradikale, die durch Kollabieren auf sich selbst oder durch Eintritt in eine Emulgatormizelle zu Partikeln nucleieren können (siehe Abschnitt 1). Ähnliche Überlegungen scheinen im Fall der genannten katalytischen Polymerisation zu stabilen Latices mit den wasserlöslichen Komplexen 7 a zuzutreffen. ${ }^{[73,79]}$ Ein alternatives Konzept zur Bildung einer großen Zahl an Latexpartikeln während der Polymerisation ist die feine Verteilung des Katalysatorvorläufers in Form einer Lösung eines hydrophoben Komplexes in Toluol/Hexadecan-Miniemulsionströpfchen (Durchmesser ca. $100 \mathrm{~nm}$ ), die in der kontinuierlichen wässerigen Phase dispergiert sind. ${ }^{[83,88]}$

Miniemulsionen ${ }^{[89-91]}$ können durch Einwirkung einer starken Scherung auf eine Mischung aus Wasser, einem Emulgator, einer organischen Phase und einem „Hydrophob“ hergestellt werden. Die starke Scherung bewirkt die Bildung von sehr kleinen Tröpfchen. Durch das Hydrophob werden diese Tröpfchen gegen die Ostwald-Reifung ${ }^{[92]}$ zu größeren Tröpfchen stabilisiert. Miniemulsionen können über lange Zeit stabil sein. Radikalische Miniemulsionspolymerisationen wurden intensiv untersucht. Es sei angemerkt, dass sich die katalytischen Polymerisationen von Ethylen, die im nächsten Absatz diskutiert werden, von der typischen radikalischen Polymerisation einer vorgebildeten Miniemulsion eines flüssigen Monomers unterscheiden (Schema 5). Gasförmiges Ethylen wird kontinuierlich der Reaktionsmischung, die zunächst aus einer Miniemulsion der Katalysatorlösung besteht, zugeführt. Die Polymerisation von Miniemulsionströpfchen des Monomers zu Partikeln derselben Größe, ein typisches Charakteristikum der Miniemulsionspolymersation im engeren Sinne, trifft also nicht zu.

Im Hinblick auf den eingesetzten Katalysator bietet die Strategie, Katalysator-Miniemulsionen für die Latexsynthese zu verwenden, zwei Vorteile: Zum einen müssen die Kataly- a)

Monomer $\quad$ starke
Wasser
Tensid
Sydrophorung
Initiator
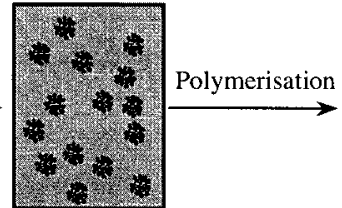

Monomer-

Miniemulsion

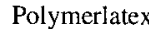
Partikel- $\varnothing$
z.B. $100 \mathrm{~nm}$

b)

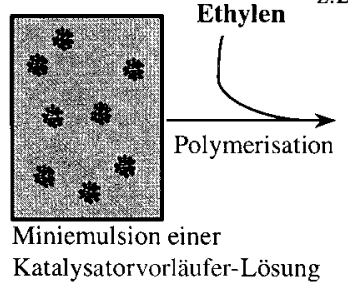

Polymerlatex

Hydrophob + Scherun

Lösungsmittel

(Kohlenwasserstoff)

Katalysatorvorläufer

Katalysatorvorläufer-Lösung

Schema 5. Vergleich zwischen einer typischen radikalischen Miniemulsionspolymerisation eines flüssigen Monomers (a) und dem speziellen Fall einer katalytischen Polymerisation von Ethylen unter Einsatz einer als Miniemulsion vorliegenden Katalysatorlösung (b).

satorvorläufer nicht hydrophil modifiziert werden, wodurch der präparative Aufwand oft verringert wird, ${ }^{[83]}$ und zum anderen können Katalysatorvorläufer mit einer gewissen Wasserempfindlichkeit verwendet werden. ${ }^{[88]}$ Mit den Katalysatoren 9 (Schema 4) haben Claverie et al. bei der Herstellung stabiler Latices aus niedermolekularem, linearem Polyethylen hohe Polymerisationsaktivitäten erzielt. ${ }^{[83]}$ Latices von 1-Olefin-Ethylen-Copolymeren wurden ebenfalls synthetisiert. ${ }^{[93]}$ Ein Vorteil der Polymerisation in Emulsion für solche Copolymerisationen ist auch folgender: In herkömmlichen Copolymerisationen von Ethylen mittels Komplexen später Übergangsmetalle werden 1-Olefine meist relativ schlecht eingebaut, und der Zusatz des Comonomers in größeren Mengen ist erforderlich. In Emulsion ermöglicht die hohe lokale Konzentration des Comonomers in den Tröpfchen einen besseren Comonomer-Einbau. Die Copolymerisation von Ethylen mit Comonomeren mit polaren, hydrophilen Substituenten ist im Zusammenhang mit Polymerlatices von besonderem Interesse. Bislang wurde über die Copolymerisation mit Undec-10-en-1-ol kurz berichtet. ${ }^{[93]}$

In den genannten Polymerisationen von Ethylen zu stabilen Latices wurden Materialien mit niedrigen Molekulargewichten erhalten. Um das Eigenschaftsprofil von Latices nutzen zu können, sind Dispersionen von Polyolefinen mit höheren Molekulargewichten erforderlich. Wir haben in unserer Gruppe Miniemulsionen des wasserempfindlichen Katalysatorvorläufers $10(\mathrm{R}=\mathrm{Me}, \mathrm{L}=$ Pyridin $)$ eingesetzt und gelangten so zu stabilen Latices von hochmolekularem, teilkristallinem Polyethylen $\left(M_{\mathrm{n}}=10^{5} \mathrm{~g} \mathrm{~mol}^{-1}, M_{\mathrm{w}} / M_{\mathrm{n}}=2-4\right) .{ }^{[88]}$

Die in den verschiedenen Emulsionspolymerisationen mit den Nickel(II)-Komplexen erhaltenen Polyethylenlatices bestehen aus Teilchen mit mittleren Durchmessern von 100 bis $600 \mathrm{~nm}$. Eine Vielzahl an anionischen Tensiden oder neutralen Stabilisatoren kann bei der Polymerisation eingesetzt werden, weil sie mit dem Katalysator verträglich sind und den Latex ausreichend stabilisieren. Feststoffgehalte bis $10 \%$ wurden bisher beschrieben. Eine typische transmissionselektronenmikroskopische (TEM-)Aufnahme zeigt Abbildung 1. Diese Partikel haben infolge ihres hohen Kristallini- 


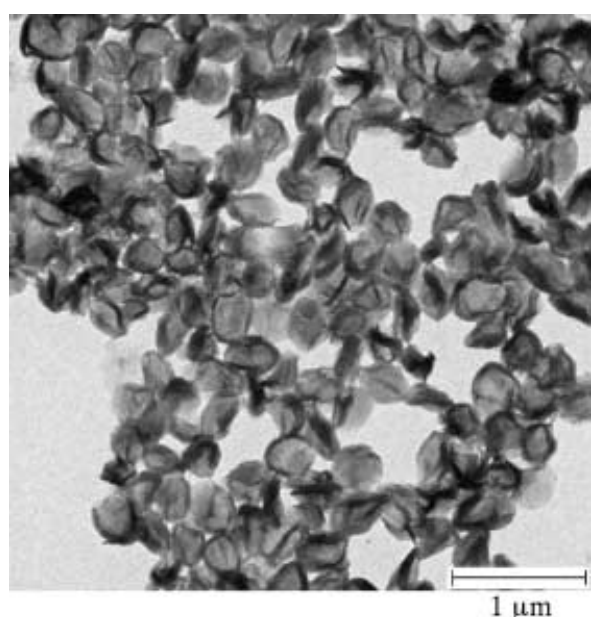

Abbildung 1. TEM-Aufnahme von durch katalytische Polymerisation hergestellten Polyethylen-Latexpartikeln $\left(M_{\mathrm{w}}=2.1 \times 10^{5} \mathrm{~g} \mathrm{~mol}^{-1}, \quad M_{\mathrm{w}} /\right.$ $M_{\mathrm{n}}=2.2$; Kristallinität: $54 \%$; Katalysatorvorläufer $10(\mathrm{R}=\mathrm{Me}, \mathrm{L}=$ Pyri$\operatorname{din})) \cdot{ }^{[88]}$

tätsgrades eine nichtsphärische Gestalt und eine rauhe Oberfläche, während die Latexpartikel von amorphem Polystyrol als gut untersuchtem Kohlenwasserstoffpolymer, das durch radikalische Emulsionspolymerisation hergestellt wird, glatt und sphärisch sind.

Vor kurzem wurde eine wässerige Olefinpolymerisation unter Verwendung eines Komplexes auf der Basis früher Übergangsmetalle beschrieben. ${ }^{\left[{ }^{[4]}\right.}$ Eine Lösung von Styrol in Toluol wurde kurz mithilfe eines Katalysators vorpolymerisiert, der durch Kombination von $\left[\left(\mathrm{C}_{5} \mathrm{Me}_{5}\right) \mathrm{Ti}(\mathrm{OMe})_{3}\right]$ mit einem Borat und einer Alkylaluminiumverbindung als Aktivator hergestellt wurde. Anschließend wurde die Reaktionsmischung in Wasser emulgiert, worauf weitere Polymerisation stattfand und syndiotaktisches Polystyrol stereoselektiv gebildet wurde. Es wird angenommen, dass der Katalysator in den emulgierten Tröpfchen enthalten und dadurch vor dem Kontakt mit Wasser geschützt ist. Die Bildung von kristallinem Polymer steigert diesen Effekt. Es wurde berichtet, dass das gebildete kristalline Polystyrol aus der Reaktionsmischung in Form relativ großer Partikel von $500 \mu \mathrm{m}$ ausfiel.

In jüngster Zeit wurde somit über mehrere wässerige katalytische Ethylenpolymerisationen berichtet. Ein besonders interessanter Aspekt ist die erfolgreiche Synthese von Latices aus weitgehend linearem Polyethylen. Die Copolymerisation mit anderen olefinischen Monomeren ermöglicht eine Steuerung der Kristallinität der Polymere. Bei der Synthese von Latices aus niedermolekularem Material wurden schon hohe Katalysatoraktivitäten erzielt. Solche Polymerisationen können als eine direkte Route zu Wachsdispersionen von Interesse sein. Auch die Zugänglichkeit von Latices aus hochmolekularem Polyethylen wurde demonstriert. Weitere Verbesserungen der Katalysatoraktivitäten sind offensichtlich ein vorrangiges Ziel. Der Einfluss geringer Mengen zugesetzter organischer Lösungsmittel, z. B. in der Miniemulsionstechnik, auf die Katalysatoraktivität bleibt zu klären. Ziegler-Katalysatoren (einschließlich Metallocenen) sind in den vergangenen 50 Jahren mit großem Aufwand optimiert worden. Verglichen mit den Aktivitäten von Metallocenen als „Single-Site“-Katalysatoren für die kom- merzielle Polyolefinproduktion $\left(>10^{7} \mathrm{TOh}^{-1}\right)$ sind die in wässrigen Ethylenpolymerisationen bislang beobachteten gering $\left(10^{5} \mathrm{TO} \mathrm{h}^{-1}\right) \cdot{ }^{[95]}$ Allerdings müssen die Katalysatoraktivitäten in wässerigen Polymerisationen nicht notwendigerweise mit denen der heutigen Polyolefinproduktion unmittelbar konkurrieren. Als eine Eigenschaft, die für eine potentielle Anwendung interessant ist, sei der im Vergleich zu StyrolButadien-Copolymeren, einem großtechnisch in einer radikalischen Emulsionspolymerisation hergestellten Kohlenwasserstoffpolymer, geringe Doppelbindungsgehalt zu nennen. Daraus kann eine beträchtlich höhere Stabilität der aus den Polymerlatices gebildeten Polymerfilme gegen UV-Licht und Luft resultieren.

\section{Polymerisation von konjugierten Dienen}

Die radikalische Polymerisation von Butadien in wässeriger Emulsion wird im großtechnischen Maßstab durchgeführt. Butadien-Styrol-Copolymerlatices werden z. B. in der Papierherstellung verwendet. ${ }^{[2]}$ Die Übergangsmetall-katalysierte Polymerisation von Dienen in nichtwässerigen Medien ist ebenfalls von großer Bedeutung. cis-1,4-Polybutadien-Gummis werden mit Ziegler-Katalysatoren auf der Basis von Titan, Cobalt, Nickel oder Neodym in Lösungsverfahren mit aromatischen Kohlenwasserstoffen als Lösungsmittel produziert. ${ }^{106]}$

Die durch $\mathrm{Rh}^{\mathrm{III}}$-Salze katalysierte Butadienpolymerisation in Wasser als Reaktionsmedium wurde schon in den 1960er Jahren von Rinehart et al. und Canale und Mitarbeitern beschrieben: Mit $\mathrm{Rh}^{\mathrm{III}} \mathrm{Cl}_{3} \cdot 3 \mathrm{H}_{2} \mathrm{O}$ als Katalysatorvorläufer lie $\beta$ sich Butadien in wässeriger Emulsion stereoselektiv $\mathrm{zu}$ teilkristallinem trans-1,4-Polybutadien polymerisieren [Gl. (2); > 99\% trans]..$^{[50,96,97]}$ Bei der radikalischen Butadien-

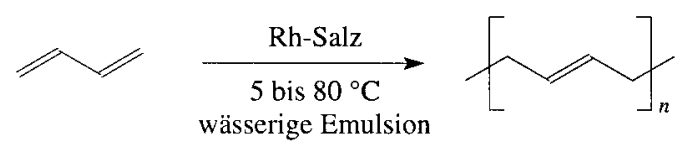

polymerisation in wässeriger Emulsion werden dagegen unter ähnlichen Bedingungen typischerweise Polymere mit ca. $60 \%$ trans-Einheiten gebildet. ${ }^{[98]}$ Bei der Butadienpolymerisation in Wasser als Reaktionsmedium mit Salzen anderer Übergangsmetalle als Rhodium wurden Polymere mit anderen Mikrostrukturen erhalten, die verfügbaren Informationen hierzu sind jedoch sehr knapp. ${ }^{[97]}$

Molekulargewichte von $2.6 \times 10^{4} \mathrm{~g} \mathrm{~mol}^{-1}$ wurden mittels Lichtstreuung bestimmt. ${ }^{[97]}$ Bei $50^{\circ} \mathrm{C}$ war die Katalysatoraktivität über $30 \mathrm{~h}$ nahezu konstant; dies zeugt von einer bemerkenswert hohen Stabilität des Katalysators in der wässerigen Polymerisation. ${ }^{[99]}$ Als geeignete Emulgatoren wurden anionische Verbindungen wie SDS oder Alkylbenzolsulfonate ermittelt. ${ }^{[96,100]}$ Aufgrund der Polymermikrostruktur und vergleichender Experimente mit Radikalinhibitoren wurde eine Koordinationspolymerisation angenommen. Eine Polymerisation nach einem radikalischen Mechanismus kann ausgeschlossen werden. Rhodium(I)-Verbindungen, die durch Reduktion der als Katalysatorvorläufer verwendeten 
$\mathrm{Rh}^{\mathrm{III}}$-Salze durch Butadien gebildet werden, wurden als aktive Spezies vorgeschlagen. In Übereinstimmung damit sind auch $\mathrm{Rh}^{\mathrm{I}}$-Komplexe wie $\left[\mathrm{Rh}_{2} \mathrm{Cl}_{2}\left(\mathrm{C}_{4} \mathrm{H}_{6}\right)_{3}\right]$ geeignete Katalysatorvorläufer mit Aktivitäten bis ca. $2 \times 10^{3} \mathrm{TOh}^{-1}$ (mit Ameisensäure als Cokatalysator). ${ }^{[101,102]}$ Anders als bei der industriell wichtigen radikalischen Copolymerisation wird Styrol bei der Rhodium-katalysierten Butadienpolymerisation nicht eingebaut. ${ }^{[103]}$ Der Rhodiumkatalysator ist auch bei Isopren $^{[101,103]}$ inaktiv, wohingegen andere Autoren über die Polymerisation von cis- oder trans-1,3-Pentadien in wässeriger Emulsion berichtet haben. ${ }^{[104]}$ Nur wenige Daten sind bezüglich der Stabilität und anderer Eigenschaften der Polymerdispersionen erhältlich. Das Ausfallen von beachtlichen Anteilen des Polymers in den Butadienpolymerisationen bei hohen Umsätzen wurde erwähnt. ${ }^{[99,105]}$

Für Dienpolymerisationen mit Komplexen später Übergangsmetalle wird im Allgemeinen angenommen, dass die wachsende Polymerkette am Metallzentrum $\eta^{3}$-Allyl-koordiniert ist (A). ${ }^{[106]}$ Im Allgemeinen sind Allylkomplexe relativ

$$
\left\langle\left(\mathrm{ML}_{n} \text { (butadien) } \mathbf{A}, \mathrm{R}=\right.\right.\text { wachsende Polymerkette }
$$

stabile Organometallverbindungen, insbesondere im Vergleich zu Alkylverbindungen. ${ }^{[107]}$ Unter diesem Aspekt mag es nicht überraschen, dass die katalytische Polymerisation von Dienen in Wasser schon verhältnismäßig früh entdeckt wurde.

Bei der Polymerisation von Butadien mit modifizierten Cobaltkatalysatoren wie dem In-situ-System [Co $\left.{ }^{\mathrm{III}}(\mathrm{acac})_{3}\right] /$ $\mathrm{AlEt} / 3 / \mathrm{H}_{2} \mathrm{O} / \mathrm{CS}_{2}$ (acac = Acetylacetonat $)$ kann hochkristallines, syndiotaktisches 1,2 -Polybutadien $\left(T_{\mathrm{m}}=205^{\circ} \mathrm{C}\right)$ erhalten werden. ${ }^{[108]}$ Die hohe Stereoselektivität dieser Katalysatoren wurde der Koordination von Schwefelkohlenstoff als Ligand am Metallzentrum zugeschrieben. ${ }^{[109]}$ Solche stereospezifischen Polymerisationen liefern in wässeriger Emulsion ${ }^{[110-112]}$ Polymerlatices [Gl. (3)].. ${ }^{[111]}$ Dabei ist eine „Vorpolymerisa-

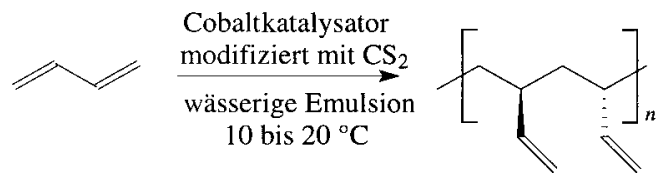

tion" mit einer kleinen Menge Butadienmonomer in Gegenwart von nur einer Spur Wasser erforderlich. Eine Lösung dieser vorpolymerisierten Katalysatormischung in einem Kohlenwasserstoff wurde anschließend in Wasser zusammen mit weiterem Butadien dispergiert und die Polymerisation fortgesetzt. Es wurde spekuliert, dass die katalytisch aktive Spezies durch das Polymer, das während der Vorpolymerisation gebildet wird, gegen den Zutritt von Wasser geschützt wird. ${ }^{[110]}$

Die genannten katalytischen Polymerisationen bieten einen Zugang zu speziellen Polymermikrostrukturen in wässeriger Emulsion. Allerdings waren insbesondere die teuren Rho- diumkomplexe, die in den ersten Arbeiten verwendet wurden, nur mäßig aktiv. Latices von Butadien-(Co)polymeren, die für eine Vielfalt von Anwendungen geeignet sind, werden schon im großen Maßstab günstig durch die etablierten radikalischen Verfahren hergestellt.

\section{Vinylische Polymerisation von cyclischen Olefinen}

Im Vergleich zur ROMP von cyclischen Olefinen (siehe Abschnitt 6) ist deren vinylische Polymerisation unter Erhaltung der Ringstruktur weniger intensiv untersucht worden. Ein frühes Beispiel einer wässerigen Polymerisation ist die Rhodium-katalysierte Polymerisation von Cyclobuten in Emulsion. Unter ähnlichen Bedingungen wie von Rinehart et al. für die Butadienpolymerisation beschrieben (Abschnitt 4) haben Natta et al. bei geringen Umsätzen kristallines, hochstereoreguläres Polymer erhalten [Gl. (4) ]. ${ }^{[13]}$ Anders als bei der Ruthenium-katalysierten wässerigen ROMP von Cyclobuten (Abschnitt 6) tritt die Ringöffnung nur als untergeordnete Nebenreaktion auf.

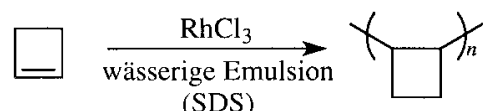

Die vinylische Polymerisation von Norbornen als dem geläufigsten Cycloolefin-Monomer kann in nichtwässerigen Medien mit Komplexen von Titan, ${ }^{[114]}$ Zirconium, ${ }^{[115]}$ Chrom, ${ }^{[116]}$ Cobalt, ${ }^{[117]}$ Nickel(II) ${ }^{[118,119]}$ oder Palladium(II) ${ }^{[120-123]}$ katalysiert werden. Späte Übergangsmetalle können hoch aktiv sein, z. B. schwach koordinierte Nickel(II)-Komplexe als Katalysatorvorläufer ${ }^{[18]}$ oder Methylalumoxan(MAO)-aktivierte Nickel(II)- und Palladium(II)-Katalysatoren. ${ }^{[124-126]}$ Die dabei erhaltenen Polynorbornene sind amorphe Kohlenwasserstoffe mit variierender Taktizität (in Abhängigkeit vom verwendeten Katalysator). Sie können eine hohe thermische Stabilität und eine geringe Dielektrizitätskonstante aufweisen. Polynorbornene und funktionalisierte Derivate werden unter dem Namen Avatrel als Materialien für elektronische Anwendungen vermarktet. ${ }^{[127,128]}$

Es sei darauf hingewiesen, dass die Reaktivität der oft als Monomer in der wässerigen Polymerisation verwendeten 2,3substituierten 5-Norbornene stark von deren Konfiguration (exo/endo) beeinflusst wird. Da diese Konfiguration in der Literatur oft nicht spezifiziert ist, ist der detaillierte Vergleich der Ergebnisse verschiedener Autoren erschwert. Außerdem wird die Charakterisierung der nichtfunktionalisierten Polynorbornene, die mit den Palladiumkatalysatoren erhalten werden, durch deren notorische Unlöslichkeit in organischen Lösungsmitteln erschwert. ${ }^{[129]}$ Die Toleranz der Norbornenpolymerisation mit $\left[\mathrm{Pd}\left(\mathrm{NCCH}_{3}\right)_{4}\right]\left(\mathrm{BF}_{4}\right)_{2}$ als Initiator gegenüber Wasser (1000 Äquiv. bezogen auf Pd) wurde von Mehler und Risse beschrieben. ${ }^{[122]}$

Über eine Polymerisation von Norbornen zu einem wässerigen Latex wurde 1993 von Perez et al. berichtet: Norbor- 
nen $\left(T_{\mathrm{m}}=44^{\circ} \mathrm{C}\right)$ wurde in wässeriger Emulsion bei $70^{\circ} \mathrm{C}$ mit SDS als Emulgator und $\mathrm{PdCl}_{2}$ als Katalysatorvorläufer umgesetzt [Gl. (5)]. ${ }^{[130,131]}$ Mit geringen Katalysatoraktivitäten von $70 \mathrm{TOh}^{-1}$ wurde ein stabiler Latex erhalten, der aus

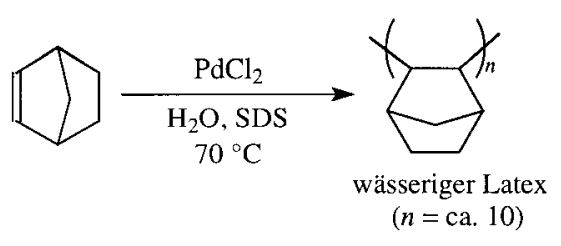

niedermolekularem, oligomerem Material $\left(\mathrm{DP}_{\mathrm{n}} \approx 10, \mathrm{DP}_{\mathrm{n}}=\right.$ Polymerisationsgrad) bestand. Interessanterweise wurde von sehr kleinen Latexpartikeln mit 10 bis $20 \mathrm{~nm}$ Durchmesser berichtet. In der radikalischen Polymerisation von olefinischen Monomeren werden solche kleinen Partikel nur durch Mikroemulsionspolymerisation erhalten. $\mathrm{PdCl}_{2}$ ist etwas wasserlöslich, und die Autoren argumentieren, dass die Polymerisation bevorzugt an der Grenzfläche zwischen Wasser und Monomertröpfchen stattfindet (obgleich die katalytisch aktive Spezies nicht eindeutig identifiziert wurde). Zur selben Zeit haben Novak et al. knapp über eine Polymerisation von polar substituierten Norbornadienen 11 a in wässeriger Emulsion mit $\mathrm{PdCl}_{2}$ berichtet [Gl. (6) ]. ${ }^{[132]}$

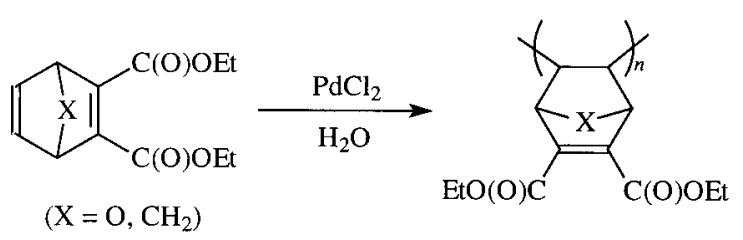

$11 \mathbf{a}$

Perez et al. haben einige Jahre später ein wasserlösliches Norbor-2-en-5-yl-substituiertes Gluconamid (11b) und ein Lactbionamid (11c) mit wasserlöslichem $\left[\mathrm{PdCl}_{2}(\mathrm{NaTPPTS})_{2}\right]$ in wässeriger Lösung polymerisiert. ${ }^{[133]}$ Mit mäßiger Effektivität (86 TO während eines gesamten Polymerisationsver-

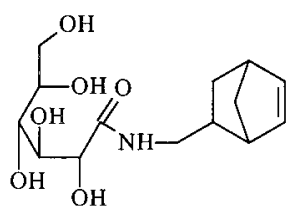

11b

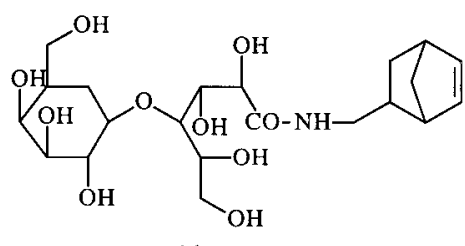

11c

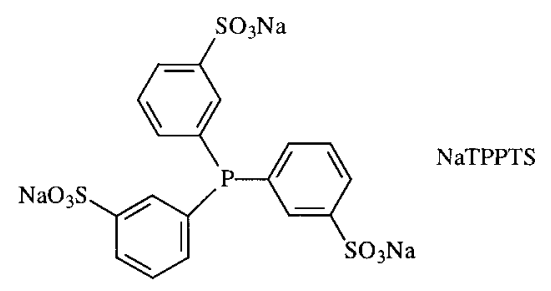

suches) wurden Oligomere mit einem Polymerisationsgrad von $\mathrm{DP}_{\mathrm{n}}=17$ bzw. 12 in einer vinylischen Polymerisation erhalten. Die wasserlöslichen Oligomere bilden in Lösung Aggregate. Wie ihre durch ROMP erhaltenen Analoga wurden solche Glycopolymere als Therapeutika vorgeschlagen. Bei der Polymerisation von [5-(Methylenammonium)norborn-2-en]chlorid mit $\mathrm{PdCl}_{2}$ in wässeriger Lösung wird ein kationischer Polyelektrolyt mit einem Polymerisationsgrad $\mathrm{DP}_{\mathrm{n}}$ von 94 erhalten. Die Zelltransfektion durch DNAKomplexe dieses Polyelektrolyts wurde untersucht. ${ }^{[134]}$

Außerdem wurde die Polymerisation von Butylnorbornen mit einem Katalysatorsystem beschrieben, das ein Äquivalent des wasserlöslichen Phosphanliganden NaTPPTS enthält $\left[\{(\text { allyl }) \mathrm{Pd}(\mu-\mathrm{Cl})\}_{2}\right] / \mathrm{NaTPPTS} / \mathrm{Li}\left[\mathrm{B}\left(\mathrm{C}_{6} \mathrm{~F}_{5}\right)_{4}\right]$. Für diese wässerige suspensionsartige Polymerisation wurde in einem Patent eine hohe Aktivität von $4.5 \times 10^{4} \mathrm{TOh}^{-1}$ beansprucht. ${ }^{[135]}$ Dies kann als ein Hinweis darauf gesehen werden, dass höhere Produktivitäten als die bisher erzielten in vinylischen wässerigen Polymerisationen von Norbornenmonomeren erreicht werden können.

\section{Ringöffnende Metathesepolymerisation}

Die ringöffnende Metathesepolymerisation (ROMP) von cyclischen Olefinen kann mit einer Vielzahl an Metallkomplex-Katalysatoren durchgeführt werden. ${ }^{[136,137]}$ Meistens werden Molybdän-, Wolfram- oder Rutheniumkatalysatoren eingesetzt. Bezüglich des Mechanismus der Olefinmetathe$\mathrm{se}^{[138]}$ besteht Einigkeit darüber, dass eine Alkylidenmetallverbindung die aktive Spezies ist (Schema 6). Durch (nichtwässerige) ROMP olefinischer Monomere wie Norbornen, Dicyclopentadien und Cycloocten hergestellte Polymere

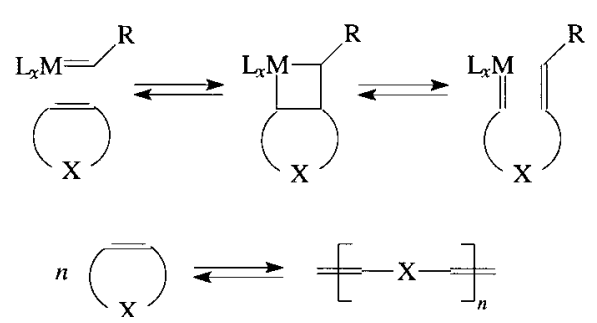

Schema 6. Mechanismus (oben) und Bruttoreaktion (unten) des Kettenwachstums durch ROMP.

werden kommerziell angewendet. ${ }^{[139]}$ In den meisten kommerziellen Verfahren und auch häufig in der Grundlagenforschung werden schlecht definierte, in situ hergestellte Katalysatoren verwendet. Der Einzug der katalytisch aktiven Alkylidenmetallkomplexe als gut definierte Katalysatorvorläufer in den 1980er Jahren war ein großer Fortschritt für die Metathese von Olefinen als Synthesemethode im Allgemeinen, und im Besonderen für die wässerige ROMP. ${ }^{[136,140-143]}$

Ein frühes Beispiel für eine wässerige ROMP wurde von Natta et al. mit der Polymerisation von Cyclobuten und 3-Methylcyclobuten unter Verwendung von $\mathrm{RuCl}_{3}$ als Katalysatorvorläufer gefunden. ${ }^{[144]}$ Mit geringen Effektivitäten von ca. 15 TO entstanden niedermolekulare, stereoirreguläre Öle. 
Die Polymerisation findet ausschließlich über Ringöffnung statt [Gl. (7)].

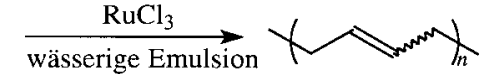

(SDS)
Rinehart et al. haben eine ROMP von Norbornen und in 5-Stellung mit Estergruppen funktionalisiertem Norborn-2-en in wässeriger Emulsion mit mäßigen Aktivitäten beschrieben. Kombinationen von Iridium(III)- oder Iridium(Iv)-Salzen mit einem Reduktionsmittel oder $\mathrm{Ir}^{\mathrm{I}}$-Olefin-Komplexe wurden als Katalysatorvorläufer eingesetzt. Auch Norbornadien und Dicyclopentadien wurden polymerisiert, wobei jeweils nur eine Doppelbindung reagierte. ${ }^{[101,145]}$

1988 publizierten Grubbs und Novak eine ROMP in wässeriger Lösung mit den funktionalisierten 7-Oxanorbornenen 12 und 13 und $\mathrm{RuCl}_{3}$ oder $\left[\mathrm{Ru}^{\mathrm{II}}\left(\mathrm{OH}_{2}\right)_{6}\right](\mathrm{OTs})_{2}$ als Katalysatorvorläufer. ${ }^{[146]}$ Die Molekulargewichte der erhalte-

tat als Carbenquelle und $\left[\mathrm{Ru}^{\mathrm{II}}\left(\mathrm{OH}_{2}\right)_{6}\right](\mathrm{OTs})_{2}$ die ROMP eines wenig reaktiven, ungespannten cyclischen Olefins wie Cycloocten möglich ist. ${ }^{[154]}$

Ein bedeutender Fortschritt war die Verwendung von isolierten Rutheniumcarbenen als Katalysatorvorläufer. Zunächst wurde festgestellt, dass Lösungen des Komplexes 17a<smiles>Cl[V](Cl)(Cl)(I)CC=C(c1ccccc1)c1ccccc1</smiles>

$\mathrm{L}=\mathrm{PPh}_{3}: \mathbf{1 7} \mathbf{a}$

$\mathrm{L}=\mathrm{PCy}_{3}: \mathbf{1 7 b}$

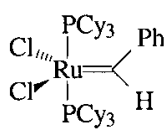

18

in organischen Medien stabil gegen den Zusatz von Wasser sind, woraus gefolgert wurde, dass solche Alkylidene als Katalysatorvorläufer für wässerige Reaktionen geeignet sein sollten. ${ }^{[155]}$ Später wurden meist Carbenkomplexe des Typs 18 statt der vinylsubstituierten Carbene des Typs $\mathbf{1 7}$ verwendet. Sie sind mit Diazoalkanen als Reagentien für die Synthese der Alkylidenmetall-Einheit einfach zugänglich und sind effizientere Initiatoren. ${ }^{[156,157]}$ Komplexe des sperrigen Trialkylphosphans $\mathrm{PCy}_{3}(\mathrm{Cy}=\mathrm{Cyclohexyl})$ zeigen im Allgemeinen höhere Aktivitäten in der ROMP als ihre $\mathrm{PPh}_{3}$-Analoga. Die Verbindungen $17 \mathbf{b}$ und 18 sind

nen Polymere waren bei gleichzeitig engeren Molekulargewichtsverteilungen $\left(M_{\mathrm{w}}=1.3 \times 10^{6} \mathrm{~g} \mathrm{~mol}^{-1} ; M_{\mathrm{w}} / M_{\mathrm{n}}=1.2\right.$ für 13) höher als bei der Polymerisation in organischen Lösungsmitteln. Die hohe Stabilität des Katalysators gegen Wasser wird durch die Tatsache unterstrichen, dass die Katalysatorlösung mehrfach genutzt werden kann. Von Vorteil ist außerdem, dass eine vorhandene „Aktivierungsphase“ des Katalysators vor der Polymerisation in Wasser weniger Zeit beansprucht als in organischen Lösungsmitteln. Eine Analyse der Polymere aus der wässerigen ROMP von 13 mit $\mathrm{RuCl}_{3}$ oder $\mathrm{OsCl}_{3}$ durch Feast und Harrison hat ergeben, dass diese Materialien ataktisch sind und ein variierendes Verhältnis von cis- zu trans-Doppelbindungen im Rückgrat aufweisen. ${ }^{[147,148]}$ Ein allgemeines Charakteristikum in der ROMP von Norbornen mit $\mathrm{RuCl}_{3}$ als Katalysatorvorläufer ist, dass bevorzugt trans-Verknüpfungen erhalten werden. Einige weitere funktionalisierte Norbornene (14-16) wurden in wässerigen Medien zu hochmolekularen Polymeren umgesetzt. ${ }^{[149-152]}$ Obgleich in den Veröffentlichungen nichts Detailliertes zu finden ist, bildeten vermutlich in allen diesen Fällen der Katalysatorvorläufer und das Monomer (12-16) zunächst eine homogene Lösung, und das wasserunlösliche Polymer fiel während der Polymerisationsreaktion aus, sofern ein weitgehend wässeriges Reaktionsmedium verwendet wurde. Acyclische Olefine

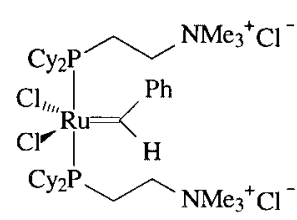

19a inzwischen kommerziell erhältlich.

Wegen der Wasserunlöslichkeit dieser Metallcarbene ist das Reaktionsmedium der wässerigen Polymerisation ein heterogenes, mehrphasiges Gemisch. Die ROMP des hydrophilen Monomers 16 oder des hydrophoben Norbornens in wässeriger Emulsion (Katalysatorvorläufer oder Initiator 17b oder 18 in Dichlormethan zugegeben) oder Suspension demonstriert, dass die Polymerisation lebend ablaufen kann. So wurde bei einem Verhältnis $\mathbf{1 6} / \mathbf{1 8}=100 / 1$ in $\mathbf{7 8} \%$ Ausbeute Poly-16 mit $M_{\mathrm{w}} / M_{\mathrm{n}}=1.07$ (gegen Polystyrolstandards) erhalten. ${ }^{[158]}$ Als Emulgator für die Emulsionspolymerisation wurde meist das kationische Dodecyltrimethylammoniumbromid (DTAB) verwendet. Außerdem sind Blockcopolymere mit engen Molekulargewichtsverteilungen zugänglich. Mit wasserlöslichen Komplexen des Typs 19 und wasserlöslichen Monomeren wie 20 kann eine lebende Polymerisation in können als Kettenüberträger fungieren und das Molekulargewicht regulieren. ${ }^{[147,149,153]}$ Die Analyse von oligomeren Produkten stützt die Annahme einer intermediären Alkylidenruthenium-Spezies. ${ }^{[153]}$ Ebenfalls in Einklang mit dem Auftreten einer solchen Spezies in der wässerigen Polymerisation ist die Beobachtung, dass mit Ethyldiazoace-<smiles></smiles>

wässeriger Lösung ohne Zusatz von Emulgatoren oder organischen Cosolventien durchgeführt werden, ${ }^{[159]}$ wobei sich die Zugabe von kleinen Mengen Säure (bis 1 Äquiv. $\mathrm{DCl})$ als vorteilhaft erwies. Es wurde nachgewiesen, dass die Säure mit dem Komplex 19a nicht an der Alkylidenmetall- 
Einheit reagiert, sondern den Monophosphankomplex und das Phosphoniumsalz des Liganden liefert. Der NMR-spektroskopische Nachweis der Alkylidenruthenium-Spezies während der Polymerisationsreaktion bestätigt deren hohe Stabilität gegen Wasser.

Über die ROMP von Norbornen in wässeriger Emulsion unter Verwendung von Ruthenium(Iv)-Komplexen ${ }^{[160]}$ mit Bis(allyl)-Liganden, wie der wasserlöslichen Verbindung $\left[\left(\eta^{3}: \eta^{3}-\mathrm{C}_{10} \mathrm{H}_{16}\right) \mathrm{Ru}\left(\mathrm{OH}_{2}\right)(\mathrm{OAc})\right] \mathrm{BF}_{4}$, als Katalysatorvorläufer berichtete Wache. ${ }^{[161]}$ Hochmolekulares Polymer mit einem ungewöhnlich hohen cis-Gehalt wurde mit einer Aktivität von $100 \mathrm{TO} \mathrm{h}^{-1}$ erhalten. Die ROMP von Deltacyclen, einem recht speziellen Monomer, in wässeriger Suspension mit $\mathrm{RuCl}_{3}$ führte zu hochmolekularem, stereoirregulärem Polymer. $^{[162]}$

Die Synthese von Neoglycopolymeren durch ROMP wurde ausführlich von Kiessling et al. untersucht (als Neoglycopolymer wird ein synthetisches Polymer mit Kohlenhydrateinheiten bezeichnet). ${ }^{[163]}$ Im Vergleich zu anderen Synthesemethoden bietet die ROMP sowohl eine gute Toleranz gegenüber funktionellen Gruppen als auch die Möglichkeit der Molekulargewichtssteuerung. Die Hydroxygruppen der Kohlenhydrat-substituierten Monomere müssen nicht geschützt werden, was den Syntheseaufwand verringert und Schwierigkeiten, die beim Entschützen auftreten können, vermeidet. Weil die Monomere und auch die gewünschten Polymere sehr hydrophil und wasserlöslich sind, kann die wässerige ROMP homogen durchgeführt werden, was für die Molekulargewichtssteuerung nützlich ist. Die ROMP von 7-Oxanorbornen mit Glucose- oder Manosesubstituenten, die über C- oder O-glycosidische Verknüpfungen gebunden sind, führt zu Neoglycopolymeren wie 21. ${ }^{[164,165]}$

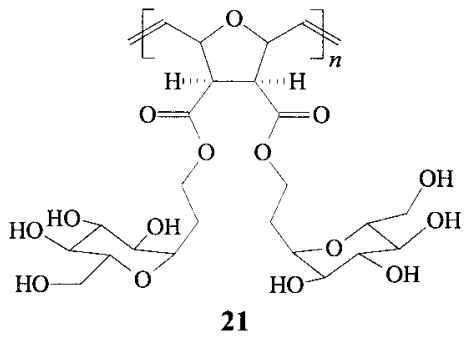

Die Reaktion kann in einer wässerigen Lösung von $\mathrm{RuCl}_{3}$ und Monomer durchgeführt werden, wobei das erhaltene Polymer ebenfalls wasserlöslich ist. Die relativen Molekulargewichte wurden mithilfe von Dextranstandards auf ca. $10^{6} \mathrm{~g} \mathrm{~mol}^{-1}$ geschätzt. Die Polymere enthalten zu annähernd gleichen Teilen cis- und trans-Doppelbindungen im Rückgrat und können durch Reduktion mit Hydrazin zu gesättigten Produkten umgesetzt werden. ${ }^{[165]}$ Die Polymerisation von Norbornenderivaten mit ungeschützten Kohlenhydratresten mithilfe der Alkylidenkomplexe $\mathbf{1 7}$ oder $\mathbf{1 8}$ als definierten Katalysatorvorläufern ist wiederum durch die Wasserunlöslichkeit dieser Rutheniumcarbene verkompliziert, kann aber in Emulsion durchgeführt werden (Wasser/Dichlormethan/ Emulgator). ${ }^{[166,167]}$ Bei der ROMP eines Carboximid-funktionalisierten Norbornens (siehe 16), das mit einer Kohlenhydrateinheit N-substituiert ist, nimmt bei Erhöhung des
Monomer/Ruthenium-Verhältnisses der durchschnittliche Polymerisationsgrad von $\mathrm{DP}_{\mathrm{n}}=10$ bis $\mathrm{DP}_{\mathrm{n}}=143 \mathrm{zu}(\mathbf{1 8}$ als Initiator). Dies weist auf eine gewisse Molekulargewichtssteuerung hin, obgleich Molekulargewichtsverteilungen nicht angegeben wurden. ${ }^{[167]}$ Die Aktivität des Proteins Concanavalin A bei der Erythrocyten-Agglutination kann durch die Bindung von Kohlenhydraten gehemmt werden. Die Struktur-Aktivitäts-Beziehung wurde unter Verwendung von Kohlenhydrat-substituierten ROMP-Polymeren mit systematisch variierter Struktur als multivalente Inhibitoren untersucht. ${ }^{[164-166,168]}$

Es sei angemerkt, dass die Entfernung von Metallresten aus mittels ROMP hergestellten ungesättigten Polymeren ein allgegenwärtiges Problem ist, ${ }^{[165,169]}$ wie schon früh von Natta et al. berichtet wurde. ${ }^{[144]}$ Es ist anzunehmen, dass die Einführung von polaren Einheiten wie Hydroxygruppen die Schwierigkeiten der Metallentfernung eher steigert. Eine auf Metallspuren beruhende geringe Stabilität des Polymers gegen Oxidation kann nachteilig sein. Auch für aussagekräftige biomedizinische Studien und im Hinblick auf potentielle biologische Anwendungen ist ein geringer Metallgehalt entscheidend. Eine Lösung dieses Problems scheint möglich zu sein, obwohl in der Literatur noch kaum detaillierte Daten vorhanden sind. ${ }^{[170,171]}$

In einigen der genannten ROMP-Experimente in Emulsion wurde eine Bildung von Latices am Rande erwähnt, jedoch ohne weitere Diskussion der Latexeigenschaften. Booth et al. berichteten über die ROMP des funktionalisierten Monomers 13 mit $\mathrm{RuCl}_{3}$ zu stabilen Dispersionen von hochmolekularem Polymer $\left(10^{5} \mathrm{~g} \mathrm{~mol}^{-1}\right.$ gegen Polystyrolstandards). ${ }^{[172]}$ Die Stabilisierung erfolgte dabei durch ein PEO- $b$-PPO- $b$-PEODreiblock-Copolymer $(\mathrm{PEO}=$ Polyethylenoxid, $\mathrm{PPO}=$ Polypropylenoxid). Während das Monomer wasserlöslich ist, ist das polymere Produkt unlöslich, und in diesem Sinne handelt es sich um eine Dispersionspolymerisation. Die erhaltenen stabilen Latices bestanden aus bemerkenswert kleinen Partikeln von z. B. 60 nm Durchmesser. Die Emulsionspolymerisation von Norbornen mit Rutheniumkatalysatoren wurde kürzlich ausführlich von Claverie et al. untersucht. ${ }^{[173]}$ Bei der Verwendung des wasserlöslichen Carbenkomplexes 19b oder des In-situ-Systems $\left.\left[\mathrm{RuCl}_{2} \text { (NaTPPTS }\right)_{2}\right] /$ Ethyldiazoacetat wurden bei $80^{\circ} \mathrm{C}$ mittlere Aktivitäten bis $10^{4} \mathrm{TOh}^{-1}$ bestimmt $^{[174]}$ (zur Struktur des wasserlöslichen Phosphans NaTPPTS siehe Abschnitt 5). Es wurden Polynorbornenlatices aus relativ kleinen Partikeln von weniger als $150 \mathrm{~nm}$ Durchmesser erhalten. Die anionischen Emulgatoren SDS oder Dowfax3B2 ${ }^{[175]}$ wurden eingesetzt, und mit letzterem wurden elektrostatisch stabilisierte Latices mit einem Feststoffgehalt bis $46 \%$ erhalten. Anders als in der typischen radikalischen Emulsionspolymerisation steigt die Partikelzahl fortlaufend mit dem Umsatz, was auf eine kontinuierliche Keimbildung hinweist. Die beobachtete Abhängigkeit der Teilchenzahl von den Reaktionsbedingungen bedeutet, dass eine homogene Keimbildung auch in Gegenwart von Emulgatormicellen auftritt. Um die Verwendung des kommerziell verfügbaren hydrophoben Carbenkomplexes $\mathbf{1 8}$ als Katalysatorvorläufer für Latexsynthesen zu ermöglichen, wurde dieser in Lösung in Form von Toluol/Hexadecan-Miniemulsionströpfchen (siehe Abschnitt 3) eingesetzt. Hierdurch konnten 
außer Norbornen auch Cycloocten und Cyclooctadien, die durch die wasserlöslichen Katalysatoren nicht polymerisiert werden, zu Latices aus hochmolekularem Polymer umgesetzt werden. Kühn et al. berichteten unabhängig davon über die Copolymerisation von Cyclopenten und Cycloocten durch $\mathbf{1 8}$ in Miniemulsion. ${ }^{[179]}$ Darüber hinaus wurde über die Herstellung von Latexpartikeln durch Copolymerisation von Norbornen und Polyethylenoxid-substituierten Norbornen-Makromonomeren mithilfe von $\mathbf{1 8}$ berichtet. ${ }^{[176]}$ Besonders bemerkenswert an dieser Reaktion, die in Ethanol/Dichlormethan durchgeführt wurde, sodass es sich nicht um eine wässerige Polymerisation handelt, ist, dass das aus der radikalischen Polymerisation bekannte Konzept der polymerisierbaren Stabilisatoren (,Surfmere“) ${ }^{[177]}$ in einer dispersionsartigen ROMP angewendet wurde.

Die Ruthenium-katalysierte wässerige ROMP hat sich somit als eine vielseitige Methode zur Synthese von polar substituierten Polymeren erwiesen. Die erhaltenen Materialien wurden auch für biomedizinische Studien verwendet. Der Einsatz von wohldefinierten Metallcarbenen als Initiatoren ermöglicht eine Steuerung der Molekulargewichte, und wasserlösliche Rutheniumcarbene sind kürzlich verfügbar geworden. In Polymerisationen von funktionalisierten Norbornenen werden typischerweise Monomer/Metall-Verhältnisse in der Größenordnung von 100/1 verwendet. Die Katalysator- oder Initiatorproduktivitäten sind im Vergleich zu denen anderer wässeriger katalytischer Polymerisationen (Abschitte 2-4) moderat, aber für die Präparation von speziellen, z. B. biologisch aktiven Materialien sind sie sicherlich zufriedenstellend. Polymerlatices wurden erfolgreich mittels wässeriger ROMP hergestellt, und der Partikelbildungsprozess wurde kürzlich untersucht.

\section{Zusammenfassung und Ausblick}

Die prinzipielle Machbarkeit der wässerigen katalytischen Polymerisation von olefinischen Monomeren wurde schon in den Pionierarbeiten der 1960er Jahre gezeigt. Signifikante Fortschritte wurden jedoch erst in den letzten Jahren erreicht: 1) Die Zahl der Polymere, die in wässerigen katalytischen Polymerisationen hergestellt wurden, ist beträchtlich gewachsen; 2) die Herstellung von stabilen wässerigen Polymerlatices wurde untersucht; 3) deutliche Verbesserungen der Katalysatoraktivität wurden erreicht. Hochmolekulare Polymere - von amorphen oder teilkristallinen Kohlenwasserstoffen bis zu polaren, wasserlöslichen Materialien - sind nun zugänglich. Der gegenwärtige Stand dieser Entwicklungen ist in Tabelle 1 zusammengefasst.

Was sind die Triebkräfte für diese Entwicklungen? Die Entdeckung von Polymerisationskatalysatoren, die auf späten Übergangsmetallen basieren, und die Verfügbarkeit von definierten Katalysatorvorläufern (die keine Alkylaluminiumverbindungen als Cokatalysatoren erfordern) haben sicherlich dazu beigetragen. Ein generell stärker werdendes Bedürfnis nach umweltfreundlichen Verfahren erhöht die Attraktivität von Wasser als Reaktionsmedium. Im Falle von Polymerlatices ist diese Umweltfreundlichkeit nicht auf das Polymerisationsverfahren beschränkt, sondern macht sich auch beim Einsatz der Produkte selbst positiv bemerkbar. Wasser ist jedoch nicht nur unter diesen Aspekten vorteilhaft, sondern ermöglicht als Polymerisationsmedium auch effiziente Verfahren. Beispielsweise macht die katalytische Polymerisation in wässeriger Emulsion Dispersionen von Polymerpartikeln von ca. $80 \mathrm{~nm}$ bis $1 \mu \mathrm{m}$ Größe, an deren Synthese und Eigenschaften großes Interesse besteht, einfach zugänglich. Außerdem ermöglicht die Toleranz der Katalysatoren gegenüber funktionellen Gruppen die Polymerisation von speziellen polaren Monomeren, wodurch Polymere z.B. für biomedizinische Studien erhalten werden - und das durch Polymerisation polarer Reaktanten in homogener Lösung.

Welche Fragen werden künftig von Interesse sein? Da die meisten mittels katalytischer Polymerisation hergestellten Latices erst in jüngster Zeit verfügbar wurden, sind noch keine Daten bezüglich anwendungsrelevanter Eigenschaften, wie der Latexstabilität unter verschiedenen Bedingungen, der

Tabelle 1. Überblick über wässerige katalytische Polymerisationen von Olefinen.

\begin{tabular}{|c|c|c|c|}
\hline Reaktionstyp & Beispiele für verwendete Katalysatorvorläufer & $\begin{array}{l}\text { typische Aktivitäten } \\
{\left[\mathrm{TO} \mathrm{h}^{-1}\right]^{[\mathrm{a}]}}\end{array}$ & Ergebnisse \\
\hline $\begin{array}{l}\text { alternierende Olefin- } \\
\text { Kohlenmonoxid- } \\
\text { Copolymerisation }\end{array}$ & kationische Pd ${ }^{\mathrm{II}}$-Diphosphankomplexe & $6 \times 10^{4}$ & $\begin{array}{l}\text { Teilkristalline Ethylen-CO-Copolymere sind mit ho- } \\
\text { hen Aktivitäten in suspensionsartigen Polymerisatio- } \\
\text { nen zugänglich. Stabile Latices von 1-Olefin-Copoly- } \\
\text { meren sind herstellbar. }\end{array}$ \\
\hline \multirow[t]{2}{*}{$\begin{array}{l}\text { Ethylen- und } \\
\text { 1-Olefinpolymerisation }\end{array}$} & neutrale $\mathrm{Ni}^{\mathrm{II}}\left(\mathrm{X}^{\frown} \mathrm{O}\right)$-Komplexe $(\mathrm{X}=\mathrm{P}, \mathrm{N})$ & $2 \times 10^{5}(\mathrm{Ni})$ & $\begin{array}{l}\text { stabile Latices von teilkristallinen Ethylen-Homo- } \\
\text { und -Copolymeren mit hohen Aktivitäten }\end{array}$ \\
\hline & kationische Pd ${ }^{\mathrm{II}}$-Diiminkomplexe & $10^{3}(\mathrm{Pd})$ & $\begin{array}{l}\text { amorphe hochverzweigte Polyethylene in suspensi- } \\
\text { onsartigen Polymerisationen }\end{array}$ \\
\hline Butadienpolymerisation & $\begin{array}{l}\text { Aluminiumalkyl-aktivierte Cobaltkatalysatoren } \\
\text { Rh-Salze }\end{array}$ & $\begin{array}{l}10^{3}(\mathrm{Co}) \\
2 \times 10^{3}(\mathrm{Rh})\end{array}$ & Latices von syndiotaktischem 1,2-Polybutadien (Co) \\
\hline $\begin{array}{l}\text { vinylische Polymerisation } \\
\text { von Cycloolefinen }\end{array}$ & $\mathrm{Pd}^{\mathrm{II}}$-Salze & ca. $10^{2}$ & $\begin{array}{l}\text { Polymerisation von Norbornen und von polar funk- } \\
\text { tionalisierten Norbornenen }\end{array}$ \\
\hline ROMP & Ru-Alkylidenkomplexe oder Ru-Salze & $\begin{array}{l}10^{4} \text { (Norbornen) } \\
10^{2} \text { (funktionalisierte } \\
\text { Norbornene) }\end{array}$ & $\begin{array}{l}\text { lebende Polymerisation von polar funktionalisierten } \\
\text { Norbornenen; kontrollierte Synthese von Polymeren } \\
\text { für biomedizinische Untersuchungen; Latices von } \\
\text { Norbornen und funktionalisierten Derivaten }\end{array}$ \\
\hline
\end{tabular}

[a] Die Aktivitäten wurden unter stark variierenden Bedingungen bestimmt und sollen deshalb nur als ein Maß für die Größenordnung verstanden werden. TO (Umsatzzahl): umgesetztes Substrat [mol] pro eingesetztem Metall [mol]. 
Filmbildung oder der Filmeigenschaften, zugänglich. Es darf erwartet werden, dass Co- und Terpolymerisation leicht verfügbarer Monomere, einschließlich polar funktionalisierter Olefine, eine Steuerung der letzteren Eigenschaften ermöglichen. Die Partikelmorphologie, z.B. die Erzeugung von Bereichen verschiedener Polarität wie in Kern-SchalePartikeln, ist ein anderer interessanter Aspekt. Hinsichtlich der Mechanismen der Partikelbildung, der Polymerisationskinetik und beispielweise bezüglich des Ortes der katalytisch aktiven Zentren während der unterschiedlichen Phasen der Polymerisation (wässerige Phase, im Monomertröpfchen oder Polymerpartikel, an den Grenzflächen) steht ein umfassendes Bild aus. Weitere Verbesserungen der Katalysatorleistung auf der Grundlage eines systematischen Verständnisses sind sicherlich erstrebenswert. Bei der Synthese eines Latex ist es wünschenswert, den Katalysator im Produkt zu belassen, um die Einfachheit dieser direkten Route zu einer Polymerdispersion zu erhalten. Daher muss die Katalysatorleistung höher sein als die Leistung $\left(<10^{3} \mathrm{TO}\right)$, die in vielen katalytischen Synthesen von niedermolekularen Verbindungen noch annehmbar ist, in denen der Katalysator zurückgewonnen wird. Allerdings müssen die Effektivitäten nicht notwendigerweise unmittelbar mit denen der Ziegler-Katalysatoren oder der Metallocene ( $\left.>10^{7} \mathrm{TO}\right)$ konkurrieren. Jüngste Entwicklungen zeigen, dass ausreichende Katalysatorleistungen prinzipiell erreicht werden können. ${ }^{[178]}$

Zusammenfassend kann festgehalten werden, dass die aktuellen Entwicklungen wässerige katalytische Polymerisationen als ein vielseitiges Thema ausweisen. Mehrere potentielle Anwendungen sind denkbar. Ebenso ist eine Anzahl attraktiver grundlegender Fragen im Bereich der Kolloidwissenschaften, der Polymerchemie, der Katalyse und der Koordinationschemie offensichtlich.

Die in dieser Übersicht zitierten Arbeiten unserer Gruppe wurden von der BASF AG und der Deutschen Forschungsgemeinschaft (SFB 428 und Graduiertenkolleg „Strukturbildung in Makromolekularen Systemen") unterstützt. S.M. dankt zudem dem Fonds der Chemischen Industrie für finanzielle Unterstützung. Wir danken den Mitgliedern der Dispersionsabteilung der BASF für fruchtbare Diskussionen. Stefan Meier (Freiburg) wird für seine Beiträge zu Abschnitt 6 gedankt.

Eingegangen am 11. Juni 2001 [A476]

[1] Emulsion Polymerization and Emulsion Polymers (Hrsg.: P. A. Lovell, M. S. El-Aasser), Wiley, Chichester, 1997.

[2] Wäßrige Polymerdispersionen (Hrsg.: D. Distler), Wiley-VCH, Weinheim, 1999.

[3] Dispersionen und Emulsionen (Hrsg.: G. Lagaly, O. Schulz, R. Zimehl), Steinkopff, Darmstadt, 1997.

[4] R. M. Fitch, Polymer Colloids: a Comprehensive Introduction, Academic Press, San Diego, 1997.

[5] a) H. U. Petereit, T. Süfke in Lit. [2], S. 277-296; b) Lit. [4], S. 164 172.

[6] S. Mecking, R. Thomann, Adv. Mater. 2000, 12, 953-956.

[7] A. M. van Herk, K. H. van Streun, J. van Welzen, A. L. German, Br. Polym. J. 1989, 21, 125-132.

[8] H. Turk, W. T. Ford, J. Org. Chem. 1991, 56, 1253-1260.

[9] „Polymer Latexes“: W. T. Ford, R. D. Badley, R. S. Chandran, S. Hari Babu, M. Hassanein, S. Srinivasan, H. Turk, H. Yu, W. Zhu, ACS Symp. Ser. 1992, 492, 423-431.
[10] Siehe beispielsweise E. Zeller, H. F. Leube, B. Schlarb, H.-J. Kneuper, M. Röper (BASF), EP 810236, 1997 [Chem. Abstr. 1998, $128,48657 \mathrm{~h}]$.

[11] Siehe beispielsweise H. F. Leube, J. Schmidt-Thümmes, E. Zeller, H. Maas (BASF), WO 99/28357, 1999 [Chem. Abstr. 1999, 131, 19491f].

[12] Anonym.

[13] F. de Dardel, T. V. Arden in Ullmann's Encyclopedia of Industrial Chemistry, Bd. A14 (Hrsg.: B. Elvers, S. Hawkins, M. Ravenscroft, G. Schulz), 5. Aufl., VCH, Weinheim, 1989, S. 393-459.

[14] Hier ist erwähnenswert, dass wasserlösliche Polymere auch als Nebenprodukt in der Emulsionspolymerisation gebildet werden können. Beispielsweise entsteht bei der Copolymerisation von Acrylsäureestern mit Acrylsäure auch wasserlösliches Polyacrylsäure-Homopolymer, das als sterischer Stabilisator für die gebildeten Latexpartikeln fungieren kann.

[15] F. L. Buchholz in Ullmann's Encyclopedia of Industrial Chemistry, Bd. A21 (Hrsg.: B. Elvers, S. Hawkins, G. Schulz), 5. Aufl., VCH, Weinheim, 1992, S. $143-156$.

[16] Ziegler Catalysts (Hrsg.: G. Fink, R. Mülhaupt, H. H. Brintzinger), Springer, Berlin, 1995.

[17] H. H. Brintzinger, D. Fischer, R. Mülhaupt, B. Rieger, R. Waymouth, Angew. Chem. 1995, 107, 1255-1283; Angew. Chem. Int. Ed. Engl. $\mathbf{1 9 9 5}, 34,1143-1170$.

[18] G. J. P. Britovsek, V. C. Gibson, D. F. Wass, Angew. Chem. 1999, 111, 448-468; Angew. Chem. Int. Ed. Engl. 1999, 38, 428-447.

[19] W. Kaminsky, M. Arndt, Adv. Polym. Sci. 1997, 127, 143-187.

[20] W. A. Herrmann in Lit. [21], S. 35-45.

[21] Aqueous-Phase Organometallic Chemistry (Hrsg.: B. Cornils, W. A. Herrmann), Wiley-VCH, Weinheim, 1998.

[22] a) W. A. Herrmann, W. C. Schattenmann in Lit. [21], S. 447-454; b) R. H. Grubbs, D. M. Lynn in Lit. [21], S. 466-476.

[23] K. Landfester, F. Tiarks, H.-P. Hentze, M. Antonietti, Macromol. Chem. Phys. 2000, 201, 1-5.

[24] J. F. Hyde, J. R. Wehrly (Dow Corning Corp.), US-Pat. 2891920, 1959 [Chem. Abstr. 1959, 53, 19449i].

[25] D. R. Weyenberg, D. E. Findlay, J. Cekada, A. E. Bey, J. Polym. Sci. Part C 1969, 27, 27-34 (ionische ringöffnende Polymerisation von cyclischen Siloxanen).

[26] C. Maitre, F. Ganachaud, O. Ferreira, J. F. Lutz, Y. Paintoux, P. Hemery, Macromolecules 2000, 33, 7730-7736, zit. Lit.

[27] K. Satoh, M. Kamigaito, M. Sawamoto, Macromolecules 1999, 32, $3827-3832$.

[28] T. I. Wallow, B. M. Novak, J. Am. Chem. Soc. 1991, 113, $7411-$ 7412.

[29] M. Rehahn, A.-D. Schlüter, G. Wegner, W. J. Feast, Polymer 1989, 30, $1060-1062$.

[30] C. Granel, P. Dubois, R. Jerome, P. Teyssie, Macromolecules 1996, 29 , $8576-8582$.

[31] S. G. Gaynor, J. Qiu, K. Matyjaszewski, Macromolecules 1998, 31, $5951-5954$.

[32] J. Storsberg, M. Hartenstein, A. H. E. Müller, H. Ritter, Macromol. Rapid Commun. 2000, 21, $1342-1346$.

[33] W. Baidossi, N. Goren, J. Blum, H. Schumann, H. Hemling, J. Mol. Catal. 1993, 85, 153-162.

[34] B. Z. Tang, W. H. Poon, S. M. Leung, W. H. Leung, H. Peng, Macromolecules 1997, 30, 2209-2212.

[35] E. Drent, P. H. M. Budzelaar, Chem. Rev. 1996, 96, 663-681.

[36] A. Sen, Acc. Chem. Res. 1993, 26, 303-310.

[37] A. S. Abu-Surrah, B. Rieger, Top. Catal. 1999, 7, 165-177.

[38] N. Alperowicz, Chem. Week 1995(2. Juli), 22.

[39] A. Gray, Chem. Br. 1998(März), 44-45.

[40] Chem. Eng. News 2000, 78(9), 16.

[41] Technische Broschüre zu Carilon von Shell.

[42] E. Drent (Shell), EP 121965, 1984 [Chem. Abstr. 1985, 102, 46423t].

[43] E. Drent, J. A. M. Van Broekhoven, M. J. Doyle, J. Organomet. Chem. 1991, 417, 235-251.

[44] E. Drent (Shell), EP 229408, 1986 [Chem. Abstr. 1988, 108, 6617b].

[45] M. Barsacchi, G. Consiglio, L. Medici, G. Petrucci, U. W. Suter, Angew. Chem. 1991, 103, 992-994; Angew. Chem. Int. Ed. Engl. 1991, 30, $989-991$.

[46] Beispiel: J. A. van Doorn, E. Drent, J. J. M. Snel (Shell), EP 280374 , 1988 [Chem. Abstr. 1989, 110, 58297h]. 
[47] J. A. van Doorn, R. L. Wife (Shell), EP 296687, 1988 [Chem. Abstr. 1989, 110, 213629p]

[48] W. Reppe, A. Magin, US-Pat. 2577208, 1951 [Chem. Abstr. 1952, 46, 6143b].

[49] Aus den in Lit. [48] veröffentlichten Daten geht nicht hervor, ob tatsächlich echte Polymere erhalten wurden. Jedoch ist deutlich, dass zumindest höhere Oligomere gebildet wurden.

[50] Für die in dieser Übersicht am häufigsten betrachteten Monomere gelten folgende Wasserlöslichkeiten $\left[\mathrm{mmol} \mathrm{L}^{-1}\right]\left(1 \mathrm{bar}, 25^{\circ} \mathrm{C}\right.$, sofern nichts Anderes angegeben): Butadien 13.6; $;^{[50 \mathrm{a}]}$ Ethylen 4.8, ${ }^{[50 \mathrm{a}]} 100$ (40 bar, $\left.25^{\circ} \mathrm{C}\right) ;^{[68]}$ Propylen $4.7 ;^{[50 \mathrm{a}]}$ Kohlenmonoxid 1.0 (1 bar, $\left.20^{\circ} \mathrm{C}\right) .^{[50 \mathrm{~b}]}$ a) CRC Handbook of Chemistry and Physics (Hrsg.: D. R. Lide), 80. Aufl., CRC, Boca Raton, USA, 1999, Kap. 8, S. $91-$ 101; b) H. Ledon in Ullmann's Encyclopedia of Industrial Chemistry, Bd. A5 (Hrsg.: W. Gerhartz), VCH, Weinheim, 1986, S. 203-216.

[51] Z. Jiang, A. Sen, Macromolecules 1994, 27, 7215-7216.

[52] G. Verspui, G. Papadogianakis, R. A. Sheldon, Chem. Commun. 1998, $401-402$.

[53] G. Verspui, F. Schanssema, R. A. Sheldon, Appl. Catal. 2000, 198, 5 11.

[54] C. Bianchini, H. Man Lee, A. Meli, S. Moneti, V. Patinec, G. Petrucci, F. Vizza, Macromolecules 1999, 32, 3859-3866.

[55] E. Lindner, M. Schmid, J. Wald, J. A. Queisser, M. Geprägs, P. Wegner, C. Nachtigal, J. Organomet. Chem. 2000, 602, 173-187.

[56] G. Verspui, F. Schanssema, R. A. Sheldon, Angew. Chem. 2000, 112, 825-827; Angew. Chem. Int. Ed. 2000, 39, 804-806; vgl. auch A. A. Broekhuis, H. Dirkzwager, H. J. Heeres, A. J. van der Linden, W. P. Mul, D. H. L. Pello, S. C. Servaas (Shell), WO 00/68296, 2000 [Chem. Abstr. 2000, 133, 350708c].

[57] M. Schmid, E. Lindner, unveröffentlichte Ergebnisse.

[58] A. Held, S. Mecking, M. Schmid, R. Pietruschka, E. Lindner, M. Khanfar, M. Sunjuk, Macromolecules, eingereicht.

[59] $\mathrm{Zu}$ Untersuchungen der Palladium-katalysierten Umsetzung von Ethylen mit CO zu Diethylketon siehe V. N. Zudin, V. D. Chinakov, V. M. Nekipelov, V. A. Rogov, V. A. Likholobov, Y. I. Yermakov, J. Mol. Catal. 1989, 52, 27-48.

[60] S. Mecking, Coord. Chem. Rev. 2000, 203, 325-351.

[61] S. D. Ittel, L. K. Johnson, M. Brookhart, Chem. Rev. 2000, 100, $1169-1204$

[62] S. Mecking, Angew. Chem. 2001, 113, 550-557; Angew. Chem. Int. Ed. 2001, 40, 534-540.

[63] H. K. Stryker, G. J. Mantell, A. F. Helin, J. Polymer Sci. Part C 1969, $27,35-48$.

[64] L. Wang, R. S. Lu, R. Bau, T. C. Flood, J. Am. Chem. Soc. 1993, 115, $6999-7000$

[65] L. K. Johnson, C. M. Killian, M. Brookhart, J. Am. Chem. Soc. 1995, 117, 6414-6415.

[66] $\mathrm{Zu}$ früheren Beobachtungen eines ähnlichen Verhaltens von neutralen Nickelkatalysatoren in 1-Olefinpolymerisationen siehe V. M. Moehring, G. Fink, Angew. Chem. 1985, 97, 982-984; Angew. Chem. Int. Ed. Engl. 1985, 24, $1001-1003$.

[67] L. K. Johnson, C. M. Killian, S. D. Arthur, J. Feldman, E. McCord, S. J. McLain, K. A. Kreutzer, M. A. Bennett, E. B. Coughlin, S. D. Ittel, A. Parthasarathy, D. Tempel, M. Brookhart (UNC-Chapel Hill/ DuPont), WO 96/23010, 1996 [Chem. Abstr. 1996, 125, 222773t].

[68] A. Held, S. Mecking, Chem. Eur. J. 2000, 6, 4623-4629.

[69] A. Held, F. Weiss, S. Mecking, Polym. Prepr. 2001, 42, 466-467.

[70] A. Held, Dissertation, Albert-Ludwigs-Universität Freiburg, 2001.

[71] Zur Diskussion hyperverzweigter Polyolefine siehe A. Sunder, J. Heinemann, H. Frey, Chem. Eur. J. 2000, 6, 2499-2506, zit. Lit.

[72] K. A. Brown, M. R. Kesti, E. Stewart, J. McGrath (3M), WO 97/ 48740, 1997 [Chem. Abstr. 1998, 128, 89246s].

[73] A. Held, F. M. Bauers, S. Mecking, Chem. Commun. 2000, 301-302.

[74] A. Tomov, J.-P. Broyer, R. Spitz, Macromol. Symp. 2000, 150, 53 58.

[75] W. Keim, F. H. Kowaldt, R. Goddard, C. Krüger, Angew. Chem. 1978, 90, 493; Angew. Chem. Int. Ed. Engl. 1978, 17, 466-467.

[76] K. A. Ostoja-Starzewski, J. Witte, Angew. Chem. 1987, 99, 76-77; Angew. Chem. Int. Ed. Engl. 1987, 26, 63-64.

[77] U. Klabunde, S. D. Ittel, J. Mol. Catal. 1987, 41, 123-134.

[78] K. Kurtev, A. Tomov, J. Mol. Catal. 1994, 88, 141-150.

[79] F. M. Bauers, S. Mecking, Macromolecules 2001, 34, 1165-1171.
[80] S. Mecking, F. M. Bauers, R. Thomann, Polym. Mater. Sci. Eng. 2001 $84,1049-1050$

[81] Die in wässeriger Emulsion mit Komplex 8 erhaltenen Polyethylene weisen eine sehr breite Molekulargewichtsverteilung auf. So wurde ein $M_{\mathrm{w}}$ bis $10^{5} \mathrm{~g} \mathrm{~mol}^{-1}$ genannt; typische Werte für $M_{\mathrm{n}}$ sind jedoch ca. $10^{3} \mathrm{~g} \mathrm{~mol}^{-1}$ : Lit. [74] und A. Tomov, R. Spitz, T. Saudemont, X. Drujon (Elf Atochem), WO 00/20464, 2000 [Chem. Abstr. 2000, 132, 279651a].

[82] Hier wäre ein systematisches Verständnis der Beziehung zwischen der Katalysatorstruktur und dem Einfluss der Ethylenkonzentration auf die Kettenwachstumsgeschwindigkeit hilfreich. Bislang existiert jedoch kein zusammenhängendes Bild. Für mehrere neutrale Nikkelkatalysatoren mit zweizähnigen Liganden wurde über eine starke Abhängigkeit der Kettenwachstumsgeschwindigkeit von der Ethylenkonzentration berichtet (in organischen Lösungsmitteln als Reaktionsmedium): a) M. Peuckert, W. Keim, Organometallics 1983, 2 , 594 - 597; b) R. H. Grubbs, Vortrag beim 219. ACS National Meeting (San Francisco), 2000, paper 230, Lit. [77] und [79]. Für sehr ähnliche Systeme ${ }^{[84]}$ wurde jedoch eine Unabhängigkeit von der Ethylenkonzentration gefunden: V. C. Gibson, A. Tomov, Polym. Mater. Sci. Eng. 2001, 84, 322-323 und entsprechender Vortrag beim 221. ACS National Meeting (San Diego), 2001. Die von Brookhart et al. untersuchte Ethylenpolymerisation mit kationischen $\mathrm{Pd}^{\mathrm{II}}$ - und $\mathrm{Ni}^{\mathrm{II}}$ Diiminkomplexen ist ebenfalls nullter Ordnung bezüglich Ethylen, die mit sehr ähnlichen kationischen Komplexen mit einer Osazoneinheit anstatt einer Aryl-Imin-Funktion dagegen erster Ordnung: P. B. Mackenzie, L. S. Moody, C. M. Killian, J. A. Ponasik, G. G. Lavoie, J. C. Pearson, T. W. Smith, L. A. Tucker, M. Moore, A. Farthing, G. King, M. Meadows, C. Sass, E. Savitski, Polym. Mater. Sci. Eng. 2001, 84, 326-327 und entsprechender Vortrag beim 221. ACS National Meeting (San Diego), 2001. Es sei zudem angemerkt, dass in vielen wässerigen Polymerisationen die lokale Katalysatorkonzentration (z.B. in Tröpfchen) relativ hoch sein kann.

[83] R. Soula, C. Novat, A. Tomov, R. Spitz, J. Claverie, X. Drujon, J. Malinge, T. Saudemont, Macromolecules 2001, 34, 2022-2026.

[84] Interessanterweise zeigte sich außerdem, dass auch die Einführung von sperrigen nichtfluorierten Substituenten die Katalysatoraktivität stark erhöht, wobei in nichtwässerigen Polymerisationen niedermolekulares Polyethylen erhalten wurde: V. C. Gibson, A. Tomov, A. J. P. White, D. J. Williams, Chem. Commun. 2001, 719-720.

[85] L. K. Johnson, A. M. A. Bennett, S. D. Ittel, L. Wang, A. Parthasarathy, E. Hauptman, R. D. Simpson, J. Feldman, E. B. Coughlin (DuPont), WO 98/30609, 1998 [Chem. Abstr. 1998, 129, 149362j].

[86] C. Wang, S. Friedrich, T. R. Younkin, R. T. Li, R. H. Grubbs, D. A Bansleben, M. W. Day, Organometallics 1998, 17, 3149-3151.

[87] T. R. Younkin, E. F. Connor, J. I. Henderson, S. K. Friedrich, R. H Grubbs, D. A. Bansleben, Science 2000, 287, 460-462.

[88] F. M. Bauers, S. Mecking, Angew. Chem. 2001, 113, 3112-3115; Angew. Chem. Int. Ed. 2001, 40, 3020-3022.

[89] P. L. Tang, E. D. Sudol, M. E. Adams, C. A. Silebi, M. S. El-Asser, ACS Symp. Ser. 1992, 492, $72-98$.

[90] E. D. Sudol, M. S. El-Asser in Lit. [1], S. 699-722.

[91] K. Landfester, Macromol. Symp. 2000, 150, 171-178.

[92] D. F. Evans, H. Wennerström, The Colloidal Domain: Where Physics, Chemistry, Biology, and Technology meet, 2. Aufl., VCH, Weinheim, 1999, S. $66-67$.

[93] J. Claverie, R. Spitz, X. Drujon, V. Touchard, M. F. Llauro, R. Soula, Polym. Mater. Sci. Eng. 2001, 84, 329-330 und entsprechender Vortrag von J. Claverie beim 221. ACS National Meeting (San Diego), 2001.

[94] B. Manders, L. Sciandrone, G. Hauck, M. O. Kristen, Angew. Chem. 2001, 113, 4139-4141; Angew. Chem. Int. Ed. 2001, 40, 40064007.

[95] Der größte Teil der Polyolefine wird mithilfe von heterogenen Ziegler-Katalysatoren produziert. Nur ein geringer Teil wird heute mit Metallocenen hergestellt. Metallocene sind für diesen Vergleich jedoch besser geeignet, da sie ebenfalls Liganden-substituierte Metallkomplexe sind.

[96] R. E. Rinehart, H. P. Smith, H. S. Witt, H. Romeyn, J. Am. Chem. Soc. 1961, 83, $4864-4865$.

[97] A. J. Canale, W. A. Hewett, T. M. Shryne, E. A. Youngman, Chem. Ind. 1962, 1054-1055. 
[98] Encyclopedia of Polymer Science and Technology, Bd. 2 (Hrsg.: H. F. Mark, N. G. Gaylord, N. M. Bikales), Wiley, New York, 1965, S. 709.

[99] R. E. Rinehart, H. P. Smith, H. S. Witt, H. Romeyn, J. Am. Chem. Soc. 1962, 84, 4145-4147.

[100] M. Morton, B. Das, J. Polym. Sci. Part C 1969, 27, 1-5.

[101] R. E. Rinehart, J. Polym. Sci. Part C 1969, 27, 7-25.

[102] In dieser Rhodium-katalysierten Polymerisation wurde eine starke pH-Abhängigkeit der Katalysatoraktivität beobachtet: Am höchsten war sie bei $\mathrm{pH} 3 ;^{[97]}$ andere Autoren haben Ameisensäure als „Cokatalysator“ verwendet. ${ }^{[101]}$

[103] P. Teyssie, R. Dauby, Polym. Lett. 1964, 2, 413-416.

[104] A. A. Entezami, R. Mechin, F. Schue, A. Collet, B. Kaempf, Eur. Polym. J. 1977, 13, 193-201.

[105] E. B. Bradford, J. W. Vanderhoff, J. Polym. Sci. Part C 1963, 3, 41-64.

[106] R. Taube, G. Sylvester in Applied Homogeneous Catalysis with Organometallic Compounds (Hrsg.: W. A. Herrmann, B. Cornils), Wiley-VCH, Weinheim, 1996, S. 280-318.

[107] Als Beispiel dafür, dass $\eta^{3}$-Allyl-Gruppen gegen protische Reagentien stabiler sind als $\eta^{1}-\mathrm{CH}_{2} \mathrm{R}-\mathrm{Gruppen}\left(\mathrm{R}=-\mathrm{CH}=\mathrm{CH}_{2}\right)$, seien die Reaktionen von $\left[\left(\eta^{1}\right.\right.$-allyl $\left.)\left(\mathrm{F}_{5} \mathrm{C}_{6}\right) \mathrm{Pd}(\mathrm{dppe})\right]$ und $\left[\left(\eta^{3}\right.\right.$-allyl $\left.)\left(\mathrm{F}_{5} \mathrm{C}_{6}\right) \mathrm{Pd}\left(\mathrm{PPh}_{3}\right)\right]$ mit $\mathrm{HCl}$ zu Propylen angeführt: H. Kurosawa, A. Urabe, K. Miki, N. Kasai, Organometallics 1986, 5, 2002-2008.

[108] H. Ashitaka, K. Jinda, H. Ueno, J. Polym. Sci. Polym. Chem. 1983, 21, $1951-1972$.

[109] H. Ashitaka, K. Jinda, H. Ueno, J. Polym. Sci. Polym. Chem. 1983, 21, $1989-1995$.

[110] J. N. Henderson, K. W. Donbar, J. J. Barbour, A. J. Bell (Goodyear), US-Pat. 4429085, 1984 [Chem Abstr. 1984, 100, 157154j].

[111] a) G. L. Burroway (Goodyear), US-Pat. 5278263, 1994 [Chem. Abstr. 1994，120，218842u]; b) G. L. Burroway, G. F. Magoun, R. N. Gujarathi (Goodyear), US-Pat. 5021381, 1991 [Chem. Abstr. 1990, $112,236729 b]$.

[112] H. Ono, T. Kato, J. Polym. Sci. Part A 2000, 38, 1083-1089.

[113] G. Natta, G. Dall'Asta, G. Motroni, Polym. Lett. 1964, 2, 349-351.

[114] G. Sartori, F. Ciampelli, N. Cameli, Chim. Ind. 1963, 45, 1478-1482.

[115] W. Kaminsky, A. Bark, M. Arndt, Macromol. Symp. 1991, 47, 83-93.

[116] U. Peucker, W. Heitz, Macromol. Rapid Commun. 1998, 19, 159162.

[117] a) F. P. Alt, W. Heitz, Macromol. Chem. Phys. 1998, 199, 1951-1956; b) B. L. Goodall, L. H. McIntosh, L. F. Rhodes, Macromol. Symp. 1995, 89, 421-432.

[118] B. L. Goodall, D. A. Barnes, G. H. Benedikt, L. H. McIntosch, Proceedings of the 6th International Business Forum on Specialty Polyolefins (SPO'96), Scotland Business Research, 1996, S. 123 142.

[119] T. J. Deming, B. M. Novak, Macromolecules 1993, 26, 7089-7091.

[120] G. Schultz, Polym. Lett. 1966, 4, 541-546.

[121] A. Sen, T.-W. Lai, Organometallics 1982, 1, 415-417.

[122] C. Mehler, W. Risse, Macromolecules 1992, 25, 4226-4228.

[123] Übersicht zur vinylischen Norbornenpolymerisation: C. Janiak, P. G. Lassahn, J. Mol. Catal. A 2001, 166, 193-209.

[124] M. Arndt, M. Gosmann, Polym. Bull. 1998, 41, 433-440.

[125] B. S. Heinz, F. P. Alt, W. Heitz, Macromol. Rapid Commun. 1998, 19 , $251-256$.

[126] C. Mast, M. Krieger, K. Dehnicke, A. Greiner, Macromol. Rapid Commun. 1999, 20, 232-235.

[127] R. A. Shick, S. K. Jayaraman, B. L. Goodall, L. F. Rhodes, W. C. McDougall, P. Kohl, S. A. Bidstrup-Allen, P. Chiniwalla, Adv. Microelectron. 1998, 25, 13-14.

[128] N. R. Grove, P. A. Kohl, S. A. Bidstrup-Allen, R. A. Shick, B. L. Goodall, S. Jayaraman, Proc. Int. Conf. Multichip Modules 1997, 6 , $224-227$.

[129] $\mathrm{Zu}$ den Eigenschaften solcher Polynorbornene und zu Berechnungen von deren Strukturen siehe auch T. A. Haselwander, W. Heitz, S. A. Krügel, J. H. Wendorff, Macromol. Chem. Phys. 1996, 197, 3435-3453.

[130] P. Eychenne, E. Perez, I. Rico, M. Bon, A. Lattes, A. Moisand, Colloid Polym. Sci. 1993, 271, 1049-1054.

[131] L. Puech, E. Perez, I. Rico-Lattes, M. Bon, A. Lattes, New J. Chem. 1997, 21, 1235-1242.

[132] A. L. Safir, B. M. Novak, Macromolecules 1993, 26, 4072-4073.

[133] L. Puech, E. Perez, I. Rico-Lattes, M. Bon, A. Lattes, A. Moisand, New J. Chem. 1997, 21, 1229-1234.
[134] E. Serres, P. Vicendo, E. Perez, T. Noel, I. Rico-Lattes, Langmuir 1999, 15, 6956-6960.

[135] J.-H. Lipian, L. F. Rhodes, B. L. Goodall, A. Bell, R. A. Mimna, J. C. Fondran, A. D. Hennis, C. N. Elia, J. D. Polley, A. Sen, J. Saikumar (BFGoodrich), WO 00/20472, 2000 [Chem. Abstr. 2000, 132, 279654d].

[136] C. Pariya, K. N. Jayaprakash, A. Sarkar, Coord. Chem. Rev. 1998 $168,1-48$.

[137] K. J. Ivin, J. C. Mol, Olefin Metathesis and Metathesis Polymerization, Academic Press, San Diego, 1997.

[138] J.-L. Hérisson, Y. Chauvin, Makromol. Chem. 1970, 141, 161-176.

[139] Beispielsweise wird Polynorbornen als synthetisches Elastomer (Norsorex, Zeon Corp.) verwendet. Amorphe Polymere, die durch Polymerisation von Norbornenderivaten (wie Dicyclopentadien) und anschließende Hydrierung der Doppelbindungen hergestell werden, werden für optische Anwendungen genutzt (Zeonex, Zeon Corp.). Polycycloocten wird als Blendkomponente in der Gummiindustrie verwendet (Vestenamer, Degussa AG). Siehe a) R. Streck in Olefin Metathesis and Polymerization Catalysts (Hrsg.: Y. Imamoglu, B. Zümreoglu-Karan, A. J. Amass), Kluwer Academic, Dordrecht, 1990, S. 439-515; b) T. Kohara, Macromol. Symp. 1996, 101 , 571-579; c) A. Iio, N. Oshima, Y. Ohira, M. Sakamoto, H. Oka (Japan Synthetic Rubber Co), DE 4139476, 1992 [Chem. Abstr. 1992, 117, 213252e].

[140] R. H. Grubbs, E. Khosravi in Material Science and Technology, Band Synthesis of Polymers (Hrsg.: R. W. Cahn, P. Haasen, E. J. Kramer, A.-D. Schlüter), Wiley-VCH, Weinheim, 1999, S. 65-104.

[141] T. M. Trnka, R. H. Grubbs, Acc. Chem. Res. 2001, 34, 18-29.

[142] R. R. Schrock, Acc. Chem. Res. 1990, 23, 158-165.

[143] M. R. Buchmeiser, Chem. Rev. 2000, 100, 1565-1604.

[144] G. Natta, G. Dall'Asta, L. Porri, Makromol. Chem. 1965, 81, 253 257.

[145] R. E. Rinehart, H. P. Smith, Polym. Lett. 1965, 3, 1049-1052.

[146] B. M. Novak, R. H. Grubbs, J. Am. Chem. Soc. 1988, 110, $7542-$ 7543

[147] W. J. Feast, D. B. Harrison, J. Mol. Catal. 1991, 65, 63-72.

[148] Siehe auch S.-Y. Lu, P. Quayle, F. Heatley, C. Booth, S. G. Yeates, J. C. Padget, Macromolecules 1992, 25, 2692-2697.

[149] W. J. Feast, D. B. Harrison, Polymer 1991, 32, 558-563.

[150] E. Zenkl, F. Stelzer, J. Mol. Catal. 1992, 76, 1-14.

[151] M. A. Hillmyer, C. Lepetit, D. V. McGrath, B. M. Novak, R. H Grubbs, Macromolecules 1992, 25, 3345-3350.

[152] Zur Polymerisation von ähnlich funktionalisierten Monomeren siehe S.-Y. Lu, J. M. Amass, N. Majid, D. Glennon, A. Byerley, F. Heatley, P. Quayle, C. Booth, Makromol. Chem. Phys. 1994, 195, 1273-1288.

[153] M. B. France, R. H. Grubbs, D. V. McGrath, R. A. Paciello, Macro molecules 1993, 26, $4742-4747$.

[154] M. B. France, R. A. Paciello, R. H. Grubbs, Macromolecules 1993 $26,4739-4741$

[155] S. T. Nguyen, L. K. Johnson, R. H. Grubbs, J. W. Ziller, J. Am. Chem. Soc. 1992, 114, 3974-3975.

[156] Einfache Wege zu vinylisch substituierten Alkylidenrutheniumkomplexen, die wiederum Vorteile gegenüber der Diazoalkanroute bieten, wurden inzwischen ebenfalls entwickelt: a) T. E. Wilhelm, T. R. Belderrain, S. N. Brown, R. H. Grubbs, Organometallics 1997, 16, 3867-3869; b) J. Wolf, W. Stüer, C. Grünwald, H. Werner, P. Schwab, M. Schulz, Angew. Chem. 1998, 110, 1165-1167; Angew. Chem. Int. Ed. 1998, 37, 1124-1126.

[157] P. Schwab, M. B. France, J. W. Ziller, R. H. Grubbs, Angew. Chem 1995, 107, 2179-2181; Angew. Chem. Int. Ed. Engl. 1995, 34, 20392041; siehe auch W. A. Herrmann, Angew. Chem. 1978, 90, 855 -868; Angew. Chem. Int. Ed. Engl. 1978, 17, 800-813.

[158] D. M. Lynn, S. Kanaoka, R. H. Grubbs, J. Am. Chem. Soc. 1996, 118 , $784-790$.

[159] a) D. M. Lynn, B. Mohr, R. H. Grubbs, J. Am. Chem. Soc. 1998, 120, 1627-1628; b) D. M. Lynn, B. Mohr, R. H. Grubbs, L. M. Henling, M. W. Day, J. Am. Chem. Soc. 2000, 122, 6601-6609.

[160] S. Wache, W. A. Herrmann, G. Artus, O. Nuyken, D. Wolf, J. Organomet. Chem. 1995, 491, 181-188.

[161] S. Wache, J. Organomet. Chem. 1995, 494, 235-240.

[162] M. Lautens, A. S. Abd-El-Aziz, J. Reibel, Macromolecules 1989, 22, $4132-4134$. 
[163] L. L. Kiessling, L. E. Strong in Topics in Organometallic Chemistry, Vol. 1 (Hrsg.: A. Fürstner), Springer, Berlin, 1998, S. 199-231.

[164] K. H. Mortell, M. Gingras, L. L. Kiessling, J. Am. Chem. Soc. 1994, 116, $12053-12054$.

[165] K. H. Mortell, R. V. Weatherman, L. L. Kiessling, J. Am. Chem. Soc. 1996, 118, 2297-2298

[166] C. Fraser, R. H. Grubbs, Macromolecules 1995, 28, 7248-7255.

[167] M. Kanai, K. H. Mortell, L. L. Kiessling, J. Am. Chem. Soc. 1997, 119, $9931-9932$.

[168] M. C. Schuster, K. H. Mortell, A. D. Hegemann, L. L. Kiessling, $J$. Mol. Catal. A 1997, 116, 209-216.

[169] A. Mühlebach, P. Bernhard, N. Bühler, T. Karlen, A. Ludi, J. Mol. Catal. 1994, 90, 143-156.

[170] Beispiel: K. Goto, Z. Komiya, N. Yamahara, A. Iio, M. Hisatomi, H. Oka (Japan Synthetic Rubber, Co), US-Pat. 5164469, 1992 [Chem. Abstr. 1989, 111, 175360e].

[171] Zur Entfernung von Ruthenium aus niedermolekularen Produkten von Metathese-Reaktionen siehe a) H. D. Maynard, R. H. Grubbs, Tetrahedron Lett. 1999, 40, 4137-4140; b) L. A. Paquette, J. D. Schloss, I. Efremov, F. Fabris, F. Gallou, J. Mendez-Andino, J. Yang, Org. Lett. 2000, 2, 1259-1261.
[172] S.-Y. Lu, P. Quayle, C. Booth, S. G. Yeates, J. C. Padget, Polymer Int. $1993,32,1-4$.

[173] J. P. Claverie, S. Viala, V. Maurel, C. Novat, Macromolecules 2001, 34, $382-388$.

[174] Diese Aktivität wurde in einem 15-min-Experiment beobachtet; siehe Lit. [173].

[175] Dowfax3B2 ist das Dinatriumsalz des disulfonierten Decyl-substituierten Diphenyloxids (Produktinformation von Dow).

[176] V. Heroguez, M. Fontanille, Y. Gnanou, Macromol. Symp. 2000, 150, $269-274$.

[177] A. Guyot, K. Tauer, Adv. Polym. Sci. 1994, 111, 43-65.

[178] Der eng verwandte Aspekt von Katalysatorresten im Polymer wurde für durch späte Übergangsmetalle katalysierte Polymerisationen intensiv diskutiert. Dieser Sensibilität für das Thema steht ein Mangel an konkreten verfügbaren Daten, z. B. zur Toxizität derartiger Reste, gegenüber. Im Detail hängt die Relevanz von Metallund Ligandenspuren wohl von der jeweiligen Anwendung und dem verwendeten Katalysator ab.

[179] I. Kühn, B. Mohr, Y. Durant, P. Schwab, R. Leyrer (BASF), DE 19859191, 2000 [Chem. Abstr. 2000, 133, 74521g]. 